\title{
Mean-square exponential stability of fuzzy stochastic BAM networks with hybrid delays
}

\author{
Fosheng Wang ${ }^{1}$ and Chengqiang Wang ${ }^{2 *}$
}

\section{"Correspondence:}

cqwung@foxmail.com

${ }^{2}$ School of Mathematics, Chengdu Normal University, Chengdu, China Full list of author information is available at the end of the article

\begin{abstract}
We study fuzzy stochastic bidirectional associative memory cellular neural networks with discrete delays in leakage terms and with continuous and infinitely distributed delays in the transmission terms. Under certain structural assumptions, we prove that the networks in question are mean-square exponentially stable. Our main ingredient is the classical direct Lyapunov approach, in which we construct an elaborate Lyapunov-Krasovskii function. The arguments in the paper can be readily adapted to study stability problems for other cellular neural networks.
\end{abstract}

MSC: Primary 93E15; secondary 28E10; 34K20; 34K37; 34K50; 60H10

Keywords: Fuzzy stochastic BAM; Mean-square stability; Hybrid delays

\section{Introduction}

Since the 1950s, various artificial neural networks (ANNs) have been designed to solve problems of pattern recognition, prediction, optimization, signal processing, associative memory, control, and so on; see, for instance, [1]. Among them, the so-called bidirectional associative memory network (BAMN) was invented and studied initially by a series of papers by Kosko [2, 3]. In the last three decades, BAMN created by Kosko was modified into the so-called fuzzy (delayed, resp. stochastic) BAMs to describe the the fuzzy transmission of information (aftereffect or memory, resp. stochastic perturbations) in the concerned BAMNs; see [4] and the vast references therein for more detail on the physical background of various BAMNs.

In recent years, deterministic BAMNs have been studied intensively and extensively from the point view of control theory and/or dynamical system theory. Gopalsamy [5] and Liu [6] studied deterministic BAMNs with delays in leakage terms for their stability. Duan and Huang [7] investigated fuzzy BAM neural networks with distributed delays and timevarying delays in the leakage terms for their global exponential stability. Cai and Huang [8] tried to understand better dynamic behaviors for memristor-based BAM neural networks with time-varying delays by utilizing theory of functional differential inclusions. Wang and Liu [9] studied a class of high-order bidirectional associative memory (BAM) neural networks with time delays in leakage terms for global exponential stability. Balasubramaniam, Kalpana, and Rakkiyappan [10] established an asymptotic stability result for BAM fuzzy cellular neural networks with time delay (discrete and unbounded distributed) in the leakage term. Li and Fan [11] considered the stability problem of almost periodic solution for Cohen-Grossberg BAM neural networks with variable coefficients. Song and Zhao

(c) The Author(s) 2018. This article is distributed under the terms of the Creative Commons Attribution 4.0 International License (http://creativecommons.org/licenses/by/4.0/), which permits unrestricted use, distribution, and reproduction in any medium, provided you give appropriate credit to the original author(s) and the source, provide a link to the Creative Commons license, and indicate if changes were made. 
[12] provided a stability criterion of complex-valued neural networks with both leakage delay and time-varying delays on time scales. Song and Cao [13] proved the exponential stability for a class of impulsive BAM neural networks with time-varying delays and reaction-diffusion terms. See [14-37] and the references therein for more information on the study of stability problems for deterministic BAMNs.

There is also a large number of references on stability and/or stabilization problems for stochastic BAMNs. Zhu, Rakkiyappan, and Chandrasekar [38] proved a stochastic stability result for Markovian jump BAM neural networks with leakage delays and impulse control. Senthilraj, Raja, Zhu, Samidurai, and Yao [39] provided the exponential passivity analysis of stochastic neural networks with leakage, distributed delays, and Markovian jumping parameters. Balasubramaniam and Vidhya [40] proved the global asymptotic stability of stochastic BAM neural networks with distributed delays and reaction-diffusion terms. Zhu, Li, and Yang [41] studied the exponential stability of stochastic reaction-diffusion BAM neural networks with time-varying and distributed delays. $\mathrm{Li}$ and $\mathrm{Fu}$ [42] provided an LMI-based stability criteria guaranteeing the global asymptotic stability for stochastic Cohen-Grossberg-type BAM neural networks with mixed delays. Bao and Cao [43] studied the exponential stability of stochastic BAM networks with discrete and distributed delays. Rakkiyappan, Chandrasekar, Lakshmanan, and Park [44] investigated a class of Markovian jumping stochastic BAM neural networks with mode-dependent probabilistic time-varying delays and impulse control for their exponential stability. Ye, Zhang, Zhang, Zhang and $\mathrm{Lu}$ [45] considered the mean-square stabilization and mean-square exponential stabilization of a class of stochastic BAM neural networks with Markovian jumping. Syed Ali, Balasubramaniam, Rihan, and Lakshmanan [46] provided a stability criteria for stochastic Takagi-Sugeno fuzzy Cohen-Grossberg BAM neural networks with mixed time-varying delays. Apart from the aforementioned references, there is still a large number of studies on the dynamics of stochastic BAMNs; see [47-54], just to name a few.

Motivated by the results obtained in the aforecited references, we are concerned in this paper with a fuzzy stochastic BAMN (see (2.1)) with discrete delays in the leakage terms, time-varying delays, and infinitely distributed delays in the drift and diffusion terms. After proving the existence of an equilibrium state, we focus on proving the mean-square exponential stability of the network under consideration. The main contribution of this work is establishing a mean-square exponential stability property for fuzzy stochastic BAM neural networks incorporating discrete delays in leakage terms and incorporating continuous and infinitely distributed delays in the transmission terms. The novelty of our proof is in employing an integral transformation to recover the dissipation mechanism hidden in the leakage terms and to design an elaborate Lyapunov-Krasovskii function to obtain the aforeclaimed mean-square exponential stability.

The rest of the paper is planed as follows. In Sect. 2, we formulate the main problem of this paper and provide some preliminaries. In Sect. 3, we state and prove in detail the main results of the paper. In Sect. 4, we provide an illustrative example for our results. In Sect. 5 , we conclude by several remarks.

\section{Formulation of the problem and preliminaries}

Let $(\Omega, \mathcal{F}, \mathbb{F}, \mathbb{P})$ be a complete filtered probability space, and let $B(t)$ be the one-dimensional standard Wiener process on this space. We assume that the filtration $\mathbb{F}=\left\{\mathcal{F}_{t} \mid t \in[0,+\infty)\right\}$ satisfies the so-called usual conditions: (i) $\mathscr{F}_{0}$ contains all $\mathbb{P}$-null sets in $\mathcal{F}$; (ii) $\mathscr{F}_{t}=\bigcap_{s>t} \mathcal{F}_{s}$. 
In the rest of the paper, $\mathbb{E} X$ denotes the mathematical expectation of a random variable $X$. Our aim in this paper is to study the following model system for BAMNs:

$$
\left\{\begin{aligned}
d u_{i}(t)= & {\left[-\mu_{1 i} u_{i}\left(t-\tau_{1 i}\right)+\sum_{j=1}^{m} a_{i j}^{1} f_{1 j}\left(v_{j}(t)\right)+\sum_{j=1}^{m} b_{i j}^{1} f_{1 j}\left(v_{j}\left(t-\sigma_{1 j}(t)\right)\right)\right.} \\
& +\bigwedge_{j=1}^{m} \alpha_{i j}^{1} \int_{-\infty}^{t} K_{1 j}(t-s) f_{1 j}\left(v_{j}(s)\right) d s \\
& +\bigvee_{j=1}^{m} \beta_{i j}^{1} \int_{-\infty}^{t} K_{1 j}(t-s) f_{1 j}\left(v_{j}(s)\right) d s \\
& \left.+\sum_{j=1}^{m} c_{i j}^{1} w_{j}^{1}+\bigwedge_{j=1}^{m} T_{i j}^{1} w_{i j}^{1}+\bigvee_{j=1}^{m} H_{i j}^{1} w_{i j}^{1}+I_{i}\right] d t \\
& +\left[\sum_{j=1}^{m} \tilde{a}_{i j} \tilde{f}_{1 j}\left(v_{j}(t)\right)+\sum_{j=1}^{m} \tilde{b}_{i j}^{1} \tilde{f}_{1 j}\left(v_{j}\left(t-\tilde{\sigma}_{1 j}(t)\right)\right)\right. \\
& +\bigwedge_{j=1}^{m} \tilde{\alpha}_{i j}^{1} \int_{-\infty}^{t} \tilde{K}_{1 j}(t-s) \tilde{f}_{1 j}\left(v_{j}(s)\right) d s \\
& \left.+\bigvee_{j=1}^{m} \tilde{\beta}_{i j}^{1} \int_{-\infty}^{t} \tilde{K}_{1 j}(t-s) \tilde{f}_{1 j}\left(v_{j}(s)\right) d s\right] d B(t), \quad i=1, \ldots, n, \\
d v_{j}(t)= & {\left[-\mu_{2 j} v_{j}\left(t-\tau_{2 j}\right)+\sum_{i=1}^{n} a_{j i}^{2} f_{2 i}\left(u_{i}(t)\right)+\sum_{i=1}^{n} b_{j i}^{2} f_{2 i}\left(u_{i}\left(t-\sigma_{2 i}(t)\right)\right)\right.} \\
& +\bigwedge_{i=1}^{n} \alpha_{j i}^{2} \int_{-\infty}^{t} K_{2 i}(t-s) f_{2 i}\left(u_{i}(s)\right) d s \\
& +\bigvee_{i=1}^{n} \beta_{j i}^{2} \int_{-\infty}^{t} K_{2 i}(t-s) f_{2 i}\left(u_{i}(s)\right) d s \\
& \left.+\sum_{i=1}^{n} c_{j i}^{2} w_{i}^{2}+\bigwedge_{i=1}^{n} T_{j i}^{2} w_{j i}^{2}+\bigvee_{i=1}^{n} H_{j i}^{2} w_{j i}^{2}+J_{j}\right] d t \\
& +\left[\sum_{i=1}^{n} \tilde{a}_{j i}^{2} \tilde{f}_{2 i}\left(u_{i}(t)\right)+\sum_{i=1}^{n} \tilde{b}_{j i}^{2} \tilde{f}_{2 i}\left(u_{i}\left(t-\tilde{\sigma}_{2 i}(t)\right)\right)\right. \\
& +\bigwedge_{i=1}^{n} \tilde{\alpha}_{j i}^{2} \int_{-\infty}^{t} \tilde{K}_{2 i}(t-s) \tilde{f}_{2 i}\left(u_{i}(s)\right) d s \\
& \left.+\bigvee_{i=1}^{n} \tilde{\beta}_{j i}^{2} \int_{-\infty}^{t} \tilde{K}_{2 i}(t-s) \tilde{f}_{2 i}\left(u_{i}(s)\right) d s\right] d B(t), \quad j=1, \ldots, m,
\end{aligned}\right.
$$

supplemented by the initial value condition

$$
u_{i}(s)=\phi_{i}(s), \quad v_{j}(s)=\psi_{j}(s), \quad \forall s \in(-\infty, 0], \mathbb{P} \text {-a.s. }
$$

where $\wedge\left(\right.$ resp. $\vee$ ) denotes the fuzzy AND (resp. OR) operation; $\mu_{1 i}>0$ and $\mu_{2 j}>0$ describe the time scales of the respective layers of the network; $\tau_{1 i}>0$ and $\tau_{2 j}>0$ denote the discrete delays in the leakage terms; the transmission coefficients $a_{i j}^{1}, a_{j i}^{2}, b_{i j}^{1}, b_{j i}^{2}, \alpha_{i j}^{1}, \alpha_{j i}^{2}, \beta_{i j}^{1}, \beta_{j i}^{2}, \tilde{a}_{i j}^{1}, \tilde{a}_{j i}^{2}$, $\tilde{b}_{i j}^{1}, \tilde{b}_{j i}^{2}, \tilde{\alpha}_{i j}^{1}, \tilde{\alpha}_{j i}^{2}, \tilde{\beta}_{i j}^{1}, \tilde{\beta}_{j i}^{2} \in \mathbb{R}$ reflect the connections of the neurons; the activation functions $f_{1 j}, \tilde{f}_{1 j}, f_{2 i}, \tilde{f}_{2 i}$ map $\mathbb{R}$ into itself; $K_{1 j}, \tilde{K}_{1 j}, K_{2 i}$, and $\tilde{K}_{2 i}$ reflect the hereditary properties of the network; $c_{i j}^{1}, c_{j i}^{2}, T_{i j}^{1}, T_{j i}^{2}, H_{i j}^{1}, H_{j i}^{2} \in \mathbb{R} ; w_{j}^{1}, w_{i}^{2}, w_{i j}^{1}, w_{j i}^{2}, I_{i}, J_{j}$ are inputs; $i=1, \ldots, n, j=1, \ldots, m$.

Assumption $1 f_{k \ell}, \tilde{f}_{k \ell}$ are Lipschitz continuous, where $\ell=1, \ldots, n$ for $k=1$, and $\ell=$ $1, \ldots, m$ for $k=2$.

Assumption $2 K_{k \ell}, \tilde{K}_{k \ell} \in L_{\text {loc }}^{1}[0,+\infty)$ take nonnegative values. There exists $\varepsilon>0$ such that $\int_{0}^{+\infty} K_{k \ell}(s) e^{\varepsilon s} d s<+\infty, \ell=1, \ldots, n$ for $k=1, \ell=1, \ldots, m$ for $k=2$.

Assumption 3 The bounded functions $\sigma_{k \ell}(t), \tilde{\sigma}_{k \ell}(t):[0,+\infty) \rightarrow[0,+\infty)$ are continuously differentiable and such that $0<\sigma_{k \ell}(t)<t, 0<\tilde{\sigma}_{k \ell}(t)<t \sup _{t \in[0,+\infty)} \dot{\sigma}_{k \ell}(t)<1$, and $\sup _{t \in[0,+\infty)} \dot{\tilde{\sigma}}_{k \ell}(t)<1, \ell=1, \ldots, n$ for $k=1, \ell=1, \ldots, m$ for $k=2$.

For convenience, we denote

$$
\begin{aligned}
\bar{\varepsilon}= & \sup \left\{\varepsilon>0 \mid \int_{0}^{+\infty} K_{1 j}(s) e^{\varepsilon s} d s, \int_{0}^{+\infty} \tilde{K}_{1 j}(s) e^{\varepsilon s} d s, \int_{0}^{+\infty} K_{2 i}(s) e^{\varepsilon s} d s,\right. \\
& \left.\int_{0}^{+\infty} \tilde{K}_{2 i}(s) e^{\varepsilon s} d s<+\infty, \quad i=1, \ldots, n, j=1, \ldots, m\right\}
\end{aligned}
$$


and

$$
\begin{array}{ll}
\check{K}_{1 j}(\varepsilon)=\int_{0}^{+\infty} K_{1 j}(s) e^{\varepsilon s} d s, & \tilde{\tilde{K}}_{1 j}(\varepsilon)=\int_{0}^{+\infty} \tilde{K}_{1 j}(s) e^{\varepsilon s} d s \\
\check{K}_{2 i}(\varepsilon)=\int_{0}^{+\infty} K_{2 i}(s) e^{\varepsilon s} d s, & \check{\tilde{K}}_{2 i}(\varepsilon)=\int_{0}^{+\infty} \tilde{K}_{2 i}(s) e^{\varepsilon s} d s \\
L_{1 j}=\sup _{u, v \in \mathbb{R}, u \neq v}\left|\frac{f_{1 j}(u)-f_{1 j}(v)}{u-v}\right|, & \tilde{L}_{1 j}=\sup _{u, v \in \mathbb{R}, u \neq v}\left|\frac{\tilde{f}_{1 j}(u)-\tilde{f}_{1 j}(v)}{u-v}\right| \\
L_{2 i}=\sup _{u, v \in \mathbb{R}, u \neq v}\left|\frac{f_{2 i}(u)-f_{2 i}(v)}{u-v}\right|, & \tilde{L}_{2 i}=\sup _{u, v \in \mathbb{R}, u \neq v}\left|\frac{\tilde{f}_{2 i}(u)-\tilde{f}_{2 i}(v)}{u-v}\right|, \\
\hat{\sigma}_{1 j}=\sup _{t \in[0,+\infty)} \dot{\sigma}_{1 j}(t), & \hat{\tilde{\sigma}}_{1 j}=\sup _{t \in[0,+\infty)} \dot{\tilde{\sigma}}_{1 j}(t) \\
\hat{\sigma}_{2 i}=\sup _{t \in[0,+\infty)} \dot{\sigma}_{2 i}(t), & \hat{\tilde{\sigma}}_{2 i}=\sup _{t \in[0,+\infty)} \dot{\tilde{\sigma}}_{2 i}(t) \\
\bar{\sigma}_{1 j}=\sup _{t \in[0,+\infty)} \sigma_{1 j}(t), & \overline{\tilde{\sigma}}_{1 j}=\sup _{t \in[0,+\infty)} \tilde{\sigma}_{1 j}(t) \\
\bar{\sigma}_{2 i}=\sup _{t \in[0,+\infty)} \sigma_{2 i}(t), & \overline{\tilde{\sigma}}_{2 i}=\sup _{t \in[0,+\infty)} \tilde{\sigma}_{2 i}(t) \\
\varepsilon \in[0, \bar{\varepsilon}), i=1, \ldots, n, j=1, \ldots, m . &
\end{array}
$$

By Assumption $1,0 \leq L_{k \ell}, \tilde{L}_{k \ell}<+\infty$; by Assumption $2,0<\bar{\varepsilon}<+\infty$, and $\check{K}_{k \ell}(\varepsilon), \check{\tilde{K}}_{k \ell}(\varepsilon)$ are increasing in $[0, \bar{\varepsilon})$; by Assumption $3,0 \leq \hat{\sigma}_{k \ell}, \hat{\tilde{\sigma}}_{k \ell}<1$. Here $\ell=1, \ldots, n$ for $k=1$, and $\ell=$ $1, \ldots, m$ for $k=2$.

We are now in a position to introduce the notion of equilibrium states. Loosely speaking, an equilibrium state of system (2.1) is any solution to the system

$$
\begin{aligned}
& -\mu_{1 i} u_{i}^{*}+\sum_{j=1}^{m} a_{i j}^{1} f_{1 j}\left(v_{j}^{*}\right)+\sum_{j=1}^{m} b_{i j}^{1} f_{1 j}\left(v_{j}^{*}\right)+\bigwedge_{j=1}^{m} \alpha_{i j}^{1} \check{K}_{1 j}(0) f_{1 j}\left(v_{j}^{*}\right) \\
& +\bigvee_{j=1}^{m} \beta_{i j}^{1} \check{K}_{1 j}(0) f_{1 j}\left(v_{j}^{*}\right)+\sum_{j=1}^{m} c_{i j}^{1} w_{j}^{1}+\bigwedge_{j=1}^{m} T_{i j}^{1} w_{i j}^{1}+\bigvee_{j=1}^{m} H_{i j}^{1} w_{i j}^{1}+I_{i}=0, \\
& \sum_{j=1}^{m} \tilde{a}_{i j}^{1} \tilde{f}_{1 j}\left(v_{j}^{*}\right)+\sum_{j=1}^{m} \tilde{b}_{i j}^{1} \tilde{f}_{1 j}\left(v_{j}^{*}\right)+\bigwedge_{j=1}^{m} \tilde{\alpha}_{i j}^{1} \check{\tilde{K}}_{1 j}(0) \tilde{f}_{1 j}\left(v_{j}^{*}\right)+\bigvee_{j=1}^{m} \tilde{\beta}_{i j}^{1} \check{\tilde{K}}_{1 j}(0) \tilde{f}_{1 j}\left(v_{j}^{*}\right)=0, \\
& -\mu_{2 j} v_{j}^{*}+\sum_{i=1}^{n} a_{j i}^{2} f_{2 i}\left(u_{i}^{*}\right)+\sum_{i=1}^{n} b_{j i}^{2} f_{2 i}\left(u_{i}^{*}\right)+\bigwedge_{i=1}^{n} \alpha_{j i}^{2} \check{K}_{2 i}(0) f_{2 i}\left(u_{i}^{*}\right) \\
& +\bigvee_{i=1}^{n} \beta_{j i}^{2} \check{K}_{2 i}(0) f_{2 i}\left(u_{i}^{*}\right)+\sum_{i=1}^{n} c_{j i}^{2} w_{i}^{2}+\bigwedge_{i=1}^{n} T_{j i}^{2} w_{j i}^{2}+\bigvee_{i=1}^{n} H_{j i}^{2} w_{j i}^{2}+J_{j}=0, \quad \text { and } \\
& n \\
& \sum_{i=1}^{n} \tilde{a}_{j i}^{2} \tilde{f}_{2 i}\left(u_{i}^{*}\right)+\sum_{i=1}^{n} \tilde{b}_{j i}^{2} \tilde{f}_{2 i}\left(u_{i}^{*}\right)+\bigwedge_{i=1}^{n} \tilde{\alpha}_{j i}^{2} \check{\tilde{K}}_{2 i}(0) \tilde{f}_{2 i}\left(u_{i}^{*}\right)+\bigvee_{i=1}^{2} \tilde{\tilde{\beta}}_{j i}^{2}(0) \tilde{f}_{2 i}\left(u_{i}^{*}\right)=0, \\
& i=1, \ldots, n, j=1, \ldots, m .
\end{aligned}
$$


Definition 1 Let $w_{j}^{1}, w_{i}^{2}, w_{i j}^{1}, w_{j i}^{2}, I_{i}, J_{j} \in L^{2}\left(\Omega, \mathcal{F}_{0}, \mathbb{P}\right), i=1, \ldots, n, j=1, \ldots, m$. Then $\left(u_{1}^{*}, \ldots\right.$, $\left.u_{n}^{*}, v_{1}^{*}, \ldots, v_{m}^{*}\right) \in L^{2}\left(\Omega, \mathcal{F}_{0}, \mathbb{P} ; \mathbb{R}^{n+m}\right)$ is said to be an equilibrium state of system (2.1) if $\left(u_{1}^{*}, \ldots, u_{n}^{*}, v_{1}^{*}, \ldots, v_{m}^{*}\right)$ is the solution to the system of equations (2.5).

Definition 2 A function $\left(u_{1}, \ldots, u_{n}, v_{1}, \ldots, v_{m}\right): \Omega \times \mathbb{R} \rightarrow \mathbb{R}^{n+m}$ is called a solution to initial value problem (IVP) (2.1)-(2.2) if $\left(u_{1}(t), \ldots, u_{n}(t), v_{1}(t), \ldots, v_{m}(t)\right)_{t \geq 0}$ is $\mathbb{F}$-adapted and $\left(u_{1}, \ldots, u_{n}, v_{1}, \ldots, v_{m}\right)$ satisfies the model (2.1) and the initial condition (2.2).

Definition 3 System (2.1) is said to be mean-square exponentially stable if every solution $\left(u_{1}, \ldots, u_{n}, v_{1}, \ldots, v_{m}\right)$ to IVP $(2.1)-(2.2)$ satisfies

$$
\begin{aligned}
& \mathbb{E}\left(\sum_{i=1}^{n}\left|u_{i}(t)-u_{i}^{*}\right|^{2}+\sum_{j=1}^{m}\left|v_{j}(t)-v_{j}^{*}\right|^{2}\right) \\
& \quad \leq \tilde{C} e^{-\tilde{\varepsilon} t} \sup _{s \in(-\infty, 0]} \mathbb{E}\left|\left(\phi_{1}(\cdot, s), \ldots, \phi_{n}(\cdot, s), \psi_{1}(\cdot, s), \ldots, \psi_{m}(\cdot, s)\right)\right|^{2}
\end{aligned}
$$

for every $t \in[0,+\infty)$ and some $\tilde{C}>0$ and $\tilde{\varepsilon}>0$, where $\left(u_{1}^{*}, \ldots, u_{n}^{*}, v_{1}^{*}, \ldots, v_{m}^{*}\right)$ is an equilibrium state of system (2.1).

Remark 2.1 Note that if $\left(u_{1}, \ldots, u_{n}, v_{1}, \ldots, v_{m}\right)$ is a solution, then

$$
\left.\left(u_{1}, \ldots, u_{n}, v_{1}, \ldots, v_{m}\right)\right|_{\Omega \times[0,+\infty)} \in L_{\mathbb{F}}^{2}\left(\Omega ; \mathcal{C}\left([0,+\infty) ; \mathbb{R}^{n+m}\right)\right) .
$$

Remark 2.2 Let Assumptions 1, 2, and 3 be fulfilled. By the theory of stochastic ODEs, for every $\left(\phi_{1}, \ldots, \phi_{n}, \psi_{1}, \ldots, \psi_{m}\right) \in \mathcal{C}\left((-\infty, 0] ; L^{2}\left(\Omega, \mathcal{F}_{0}, \mathbb{P} ; \mathbb{R}^{n+m}\right)\right)$ satisfying $\left(\right.$ a) $\left(\phi_{1}(\omega, \cdot), \ldots\right.$, $\left.\phi_{n}(\omega, \cdot), \psi_{1}(\omega, \cdot), \ldots, \psi_{m}(\omega, \cdot)\right)$ is continuous on $(-\infty, 0], \mathbb{P}$-a.s., and $(\mathrm{b}) \sup _{s \in(-\infty, 0]} \mathbb{E} \mid\left(\phi_{1}(\cdot, s)\right.$, $\left.\ldots, \phi_{n}(\cdot, s), \psi_{1}(\cdot, s), \ldots, \psi_{m}(\cdot, s)\right)\left.\right|^{2}<+\infty, \operatorname{IVP}(2.1)-(2.2)$ admits a unique solution.

Remark 2.3 If system (2.1) is mean-square exponentially stable, then it has a unique fixed point.

We conclude this section by including two necessary lemmas. In the first lemma, we present the well-known Gronwall's inequality, and in the second lemma, we collect two facts about the fuzzy AND and OR operations. Since the proofs are not rare in the literature, we omit their details here.

Lemma 2.1 (Gronwall's lemma) Let $\delta \in \mathbb{R}$, let $x:\left[t_{0}, T\right] \rightarrow \mathbb{R}$ be bounded, and let $h$ : $\left[t_{0}, T\right] \rightarrow[0,+\infty)$ be Lebesgue integrable. If $x(t) \leq \delta+\int_{t_{0}}^{t} h(s) x(s) d s$ for $t \in\left[t_{0}, T\right]$, then $x(t) \leq \delta e^{\int_{t_{0}}^{t} h(s) d s}$ for all $t \in\left[t_{0}, T\right]$.

Lemma 2.2 For all $x=\left(x_{1}, \ldots, x_{N}\right)^{\top}, y=\left(y_{1}, \ldots, y_{N}\right)^{\top} \in \mathbb{R}^{N}$, and $\left(\mu_{1}, \ldots, \mu_{N}\right)^{\top} \in \mathbb{R}^{N}$, we have $\left|\bigvee_{k=1}^{N} \mu_{k} x_{k}-\bigvee_{k=1}^{N} \mu_{k} y_{k}\right| \leq \sum_{k=1}^{N}\left|\mu_{k}\right|\left|x_{k}-y_{k}\right|$ and $\left|\bigwedge_{k=1}^{N} \mu_{k} x_{k}-\bigwedge_{k=1}^{N} \mu_{k} y_{k}\right| \leq$ $\sum_{k=1}^{N}\left|\mu_{k}\right|\left|x_{k}-y_{k}\right|$.

\section{Main results and their proofs}

In this section, we prove under some conditions that system (2.1) has a unique equilibrium state by Banach's contraction fixed point argument and prove under some additional 
conditions that system (2.1) is mean-square exponentially stable. The main ingredient in proving the stability result is a well-chosen Lyapunov functional. We first state the first main result concerned with the existence of equilibrium states of system (2.1).

Theorem 3.1 Let Assumptions 1, 2, and 3 be fulfilled. Suppose in addition that the following two properties hold:

(i) If $\left(u_{1}, \ldots, u_{n}, v_{1}, \ldots, v_{m}\right)$ solves the system

$$
\left\{\begin{aligned}
\mu_{1 i} u_{i}= & \sum_{j=1}^{m} a_{i j}^{1} f_{1 j}\left(v_{j}\right)+\sum_{j=1}^{m} b_{i j}^{1} f_{1 j}\left(v_{j}\right)+\bigwedge_{j=1}^{m} \alpha_{i j}^{1} \check{K}_{1 j}(0) f_{1 j}\left(v_{j}\right) \\
& +\bigvee_{j=1}^{m} \beta_{i j}^{1} \check{K}_{1 j}(0) f_{1 j}\left(v_{j}\right)+\sum_{j=1}^{m} c_{i j}^{1} w_{j}^{1}+\bigwedge_{j=1}^{m} T_{i j}^{1} w_{i j}^{1}+\bigvee_{j=1}^{m} H_{i j}^{1} w_{i j}^{1}+I_{i}, \\
\mu_{2 j} v_{j}= & \sum_{i=1}^{n} a_{j i}^{2} f_{2 i}\left(u_{i}\right)+\sum_{i=1}^{n} b_{j i}^{2} f_{2 i}\left(u_{i}\right)+\bigwedge_{i=1}^{n} \alpha_{j i}^{2} \check{K}_{2 i}(0) f_{2 i}\left(u_{i}\right) \\
& +\bigvee_{i=1}^{n} \beta_{j i}^{2} \check{K}_{2 i}(0) f_{2 i}\left(u_{i}\right)+\sum_{i=1}^{n} c_{j i}^{2} w_{i}^{2}+\bigwedge_{i=1}^{n} T_{j i}^{2} w_{j i}^{2}+\bigvee_{i=1}^{n} H_{j i}^{2} w_{j i}^{2}+J_{j},
\end{aligned}\right.
$$

then $\left(u_{1}, \ldots, u_{n}, v_{1}, \ldots, v_{m}\right)$ satisfies

$$
\left\{\begin{array}{l}
\sum_{j=1}^{m} \tilde{a}_{i j}^{1} \tilde{f}_{1 j}\left(v_{j}\right)+\sum_{j=1}^{m} \tilde{b}_{i j}^{1} \tilde{f}_{1 j}\left(v_{j}\right)+\bigwedge_{j=1}^{m} \tilde{\alpha}_{i j}^{1} \check{\tilde{K}}_{1 j}(0) \tilde{f}_{1 j}\left(v_{j}\right)+\bigvee_{j=1}^{m} \tilde{\beta}_{i j}^{1} \check{\tilde{K}}_{1 j}(0) \tilde{f}_{1 j}\left(v_{j}\right)=0, \\
\sum_{i=1}^{n} \tilde{a}_{j i}^{2} \tilde{f}_{2 i}\left(u_{i}\right)+\sum_{i=1}^{n} \tilde{b}_{j i}^{2} \tilde{f}_{2 i}\left(u_{i}\right)+\bigwedge_{i=1}^{n} \tilde{\alpha}_{j i}^{2} \tilde{\tilde{K}}_{2 i}(0) \tilde{f}_{2 i}\left(u_{i}\right)+\bigvee_{i=1}^{n} \tilde{\beta}_{j i}^{2} \tilde{\tilde{K}}_{2 i}(0) \tilde{f}_{2 i}\left(u_{i}\right)=0 ;
\end{array}\right.
$$

(ii) $\lambda_{1}<1$ and $\lambda_{2}<1$ with

$$
\left.\begin{array}{l}
\lambda_{1}=\max _{1 \leq j \leq m} \sum_{i=1}^{n} \frac{L_{1 j}\left(\left|a_{i j}^{1}\right|+\left|b_{i j}^{1}\right|+\breve{K}_{1 j}(0)\left|\alpha_{i j}^{1}\right|+\check{K}_{1 j}(0)\left|\beta_{i j}^{1}\right|\right)}{\mu_{1 i}}, \\
\lambda_{2}=\max _{1 \leq i \leq n} \sum_{j=1}^{m} \frac{L_{2 i}\left(\left|a_{j i}^{2}\right|+\left|b_{j i}^{2}\right|+\check{K}_{2 i}(0)\left|\alpha_{j i}^{2}\right|+\check{K}_{2 i}(0)\left|\beta_{j i}^{2}\right|\right)}{\mu_{2 j}}
\end{array}\right\}
$$

Then system (2.1) admits a unique equilibrium state.

Proof Let us define the nonlinear mapping on $\mathbb{R}^{n+m}$ by

$$
\begin{aligned}
\Psi\left(u_{1}, \ldots, u_{n}, v_{1}, \ldots, v_{m}\right) & \left(\sum_{j=1}^{m} \frac{a_{1 j}^{1} f_{1 j}\left(v_{j}\right)}{\mu_{11}}+\sum_{j=1}^{m} \frac{b_{1 j}^{1} f_{1 j}\left(v_{j}\right)}{\mu_{11}}+\bigwedge_{j=1}^{m} \frac{\alpha_{1 j}^{1} \check{K}_{1 j}(0) f_{1 j}\left(v_{j}\right)}{\mu_{11}}+\bigvee_{j=1}^{m} \frac{\beta_{1 j}^{1} \check{K}_{1 j}(0) f_{1 j}\left(v_{j}\right)}{\mu_{11}}\right. \\
& +\sum_{j=1}^{m} \frac{c_{1 j}^{1} w_{j}^{1}}{\mu_{11}}+\bigwedge_{j=1}^{m} \frac{T_{1 j}^{1} w_{1 j}^{1}}{\mu_{11}}+\bigvee_{j=1}^{m} \frac{H_{1 j}^{1} w_{1 j}^{1}}{\mu_{11}}+\frac{I_{1}}{\mu_{11}}, \ldots, \sum_{j=1}^{m} \frac{a_{n j}^{1} f_{1 j}\left(v_{j}\right)}{\mu_{1 n}}+\sum_{j=1}^{m} \frac{b_{n j}^{1} f_{1 j}\left(v_{j}\right)}{\mu_{1 n}} \\
& +\bigwedge_{j=1}^{m} \frac{\alpha_{n j}^{1} \check{K}_{1 j}(0) f_{1 j}\left(v_{j}\right)}{\mu_{1 n}}+\bigvee_{j=1}^{m} \frac{\beta_{n j}^{1} \check{K}_{1 j}(0) f_{1 j}\left(v_{j}\right)}{\mu_{1 n}}+\sum_{j=1}^{m} \frac{c_{n j}^{1} w_{j}^{1}}{\mu_{1 n}}+\bigwedge_{j=1}^{m} \frac{T_{n j}^{1} w_{n j}^{1}}{\mu_{1 n}} \\
& +\bigvee_{j=1}^{m} \frac{H_{n j}^{1} w_{n j}^{1}}{\mu_{1 n}}+\frac{I_{n}}{\mu_{1 n}}, \sum_{i=1}^{n} \frac{a_{11}^{2} f_{2 i}\left(u_{i}\right)}{\mu_{21}}+\sum_{i=1}^{n} \frac{b_{1 i}^{2} f_{2 i}\left(u_{i}\right)}{\mu_{21}}+\bigwedge_{i=1}^{n} \frac{\alpha_{1 i}^{2} \check{K}_{2 i}(0) f_{2 i}\left(u_{i}\right)}{\mu_{21}} \\
& +\bigvee_{i=1}^{n} \frac{\beta_{1 i}^{2} \check{K}_{2 i}(0) f_{2 i}\left(u_{i}\right)}{\mu_{21}}+\sum_{i=1}^{n} \frac{c_{1 i}^{2} w_{i}^{2}}{\mu_{21}}+\bigwedge_{i=1}^{n} \frac{T_{1 i}^{2} w_{1 i}^{2}}{\mu_{21}}+\bigvee_{i=1}^{n} \frac{H_{1 i}^{2} w_{1 i}^{2}}{\mu_{21}}+\frac{J_{1}}{\mu_{21}}, \ldots, \\
& \sum_{i=1}^{n} \frac{a_{m i}^{2} f_{2 i}\left(u_{i}\right)}{\mu_{2 m}}+\sum_{i=1}^{n} \frac{b_{m i}^{2} f_{2 i}\left(u_{i}\right)}{\mu_{2 m}}+\bigwedge_{i=1}^{n} \frac{\alpha_{m i}^{2} \check{K}_{2 i}(0) f_{2 i}\left(u_{i}\right)}{\mu_{2 m}}+\bigvee_{i=1}^{n} \frac{\beta_{m i}^{2} \check{K}_{2 i}(0) f_{2 i}\left(u_{i}\right)}{\mu_{2 m}}
\end{aligned}
$$




$$
\left.+\sum_{i=1}^{n} \frac{c_{m i}^{2} w_{i}^{2}}{\mu_{2 m}}+\bigwedge_{i=1}^{n} \frac{T_{m i}^{2} w_{m i}^{2}}{\mu_{2 m}}+\bigvee_{j=1}^{m} \frac{H_{m i}^{2} w_{m i}^{2}}{\mu_{2 m}}+\frac{J_{m}}{\mu_{2 m}}\right)
$$

for $\left(u_{1}, \ldots, u_{n}, v_{1}, \ldots, v_{m}\right) \in \mathbb{R}^{n+m}$.

For every $X_{1} \in \mathbb{R}^{n+m}$ and every $X_{2} \in \mathbb{R}^{n+m}$ with $X_{1}=\left(u_{1}, \ldots, u_{n}, v_{1}, \ldots, v_{m}\right)$ and $X_{2}=$ $\left(x_{1}, \ldots, x_{n}, y_{1} \ldots, y_{m}\right)$, by Lemma 2.2 and by conducting some routine calculations we obtain the following sequence of inequalities:

$$
\begin{aligned}
& \left\|\Psi\left(X_{1}\right)-\Psi\left(X_{2}\right)\right\| \\
& =\sum_{i=1}^{n} \mid \sum_{j=1}^{m} \frac{a_{i j}^{1} f_{1 j}\left(v_{j}\right)}{\mu_{1 i}}+\sum_{j=1}^{m} \frac{b_{i j}^{1} f_{1 j}\left(v_{j}\right)}{\mu_{1 i}}+\bigwedge_{j=1}^{m} \frac{\alpha_{i j}^{1} \breve{K}_{1 j}(0) f_{1 j}\left(v_{j}\right)}{\mu_{1 i}}+\bigvee_{j=1}^{m} \frac{\beta_{i j}^{1} \breve{K}_{1 j}(0) f_{1 j}\left(v_{j}\right)}{\mu_{1 i}} \\
& -\sum_{j=1}^{m} \frac{a_{i j}^{1} f_{1 j}\left(y_{j}\right)}{\mu_{1 i}}-\sum_{j=1}^{m} \frac{b_{i j}^{1} f_{1 j}\left(y_{j}\right)}{\mu_{1 i}}-\bigwedge_{j=1}^{m} \frac{\alpha_{i j}^{1} \check{K}_{1 j}(0) f_{1 j}\left(y_{j}\right)}{\mu_{1 i}}-\bigvee_{j=1}^{m} \frac{\beta_{i j}^{1} \check{K}_{1 j}(0) f_{1 j}\left(y_{j}\right)}{\mu_{1 i}} \mid \\
& +\sum_{j=1}^{m} \mid \sum_{i=1}^{n} \frac{a_{j i}^{2} f_{2 i}\left(u_{i}\right)}{\mu_{2 j}}+\sum_{i=1}^{n} \frac{b_{j i}^{2} f_{2 i}\left(u_{i}\right)}{\mu_{2 j}}+\bigwedge_{i=1}^{n} \frac{\alpha_{j i}^{2} \check{K}_{2 i}(0) f_{2 i}\left(u_{i}\right)}{\mu_{2 j}}+\bigvee_{i=1}^{n} \frac{\beta_{j i}^{2} \breve{K}_{2 i}(0) f_{2 i}\left(u_{i}\right)}{\mu_{2 j}} \\
& -\sum_{i=1}^{n} \frac{a_{j i}^{2} f_{2 i}\left(x_{i}\right)}{\mu_{2 j}}-\sum_{i=1}^{n} \frac{b_{j i}^{2} f_{2 i}\left(x_{i}\right)}{\mu_{2 j}}-\bigwedge_{i=1}^{n} \frac{\alpha_{j i}^{2} \check{K}_{2 i}(0) f_{2 i}\left(x_{i}\right)}{\mu_{2 j}}-\bigvee_{i=1}^{n} \frac{\beta_{j i}^{2} \check{K}_{2 i}(0) f_{2 i}\left(x_{i}\right)}{\mu_{2 j}} \\
& \leq \sum_{i=1}^{n}\left[\left|\sum_{j=1}^{m} \frac{a_{i j}^{1} f_{1 j}\left(v_{j}\right)}{\mu_{1 i}}-\sum_{j=1}^{m} \frac{a_{i j}^{1} f_{1 j}\left(y_{j}\right)}{\mu_{1 i}}\right|+\left|\sum_{j=1}^{m} \frac{b_{i j}^{1} f_{1 j}\left(v_{j}\right)}{\mu_{1 i}}-\sum_{j=1}^{m} \frac{b_{i j}^{1} f_{1 j}\left(y_{j}\right)}{\mu_{1 i}}\right|\right. \\
& +\left|\bigwedge_{j=1}^{m} \frac{\alpha_{i j}^{1} \check{K}_{1 j}(0) f_{1 j}\left(v_{j}\right)}{\mu_{1 i}}-\bigwedge_{j=1}^{m} \frac{\alpha_{i j}^{1} \check{K}_{1 j}(0) f_{1 j}\left(y_{j}\right)}{\mu_{1 i}}\right| \\
& \left.+\left|\bigvee_{j=1}^{m} \frac{\beta_{i j}^{1} \check{K}_{1 j}(0) f_{1 j}\left(v_{j}\right)}{\mu_{1 i}}-\bigvee_{j=1}^{m} \frac{\beta_{i j}^{1} \check{K}_{1 j}(0) f_{1 j}\left(y_{j}\right)}{\mu_{1 i}}\right|\right] \\
& +\sum_{j=1}^{m}\left[\left|\sum_{i=1}^{n} \frac{a_{j i}^{2} f_{2 i}\left(u_{i}\right)}{\mu_{2 j}}-\sum_{i=1}^{n} \frac{a_{j i}^{2} f_{2 i}\left(u_{i}\right)}{\mu_{2 j}}\right|+\left|\sum_{i=1}^{n} \frac{b_{j i}^{2} f_{2 i}\left(u_{i}\right)}{\mu_{2 j}}-\sum_{i=1}^{n} \frac{b_{j i}^{2} f_{2 i}\left(u_{i}\right)}{\mu_{2 j}}\right|\right. \\
& +\left|\bigwedge_{i=1}^{n} \frac{\alpha_{j i}^{2} \check{K}_{2 i}(0) f_{2 i}\left(u_{i}\right)}{\mu_{2 j}}-\bigwedge_{i=1}^{n} \frac{\alpha_{j i}^{2} \check{K}_{2 i}(0) f_{2 i}\left(x_{i}\right)}{\mu_{2 j}}\right| \\
& \left.+\left|\bigvee_{i=1}^{n} \frac{\beta_{j i}^{2} \check{K}_{2 i}(0) f_{2 i}\left(u_{i}\right)}{\mu_{2 j}}-\bigvee_{i=1}^{n} \frac{\beta_{j i}^{2} \check{K}_{2 i}(0) f_{2 i}\left(x_{i}\right)}{\mu_{2 j}}\right|\right] \\
& \leq \lambda_{1} \sum_{j=1}^{m}\left|v_{j}-y_{j}\right|+\lambda_{2} \sum_{i=1}^{n}\left|u_{i}-x_{i}\right| \leq \max \left(\lambda_{1}, \lambda_{2}\right)\left\|X_{1}-X_{2}\right\|,
\end{aligned}
$$

where $\lambda_{1}$ and $\lambda_{2}$ are given by (3.1). Since $\lambda_{1}<1$ and $\lambda_{2}<1$ by assumption, this means that $\Psi$ is a (strict) contraction on $\mathbb{R}^{n+m}$. By Banach's contraction fixed point argument, $\Psi$ admits a unique fixed point $\left(u_{1}^{*}, \ldots, u_{n}^{*}, v_{1}^{*}, \ldots, v_{m}^{*}\right)$ in $\mathbb{R}^{n+m}$. By the definition of $\Psi$, $\left(u_{1}^{*}, \ldots, u_{n}^{*}, v_{1}^{*}, \ldots, v_{m}^{*}\right)$ is indeed an equilibrium state of system (2.1).

The uniqueness can be obtained readily, since $\Psi$ is a strict contraction. The proof is complete. 
Theorem 3.2 Let Assumptions 1, 2, and 3 be fulfilled. If $M_{1 i}(0)<0, M_{2 j}(0)<0(i=1, \ldots, n$, $j=1, \ldots, m)$, and the hypothesis of Theorem 3.1 are satisfied, then system (2.1) is meansquare exponentially stable. Here $M_{1 i}$ and $M_{2 j}$ are continuous functions on $[0, \bar{\varepsilon})$ given by

$$
\begin{aligned}
M_{1 i}(\varepsilon)= & -2\left(\mu_{1 i} e^{\varepsilon \tau_{1 i}}-\varepsilon\right)+\sum_{j=1}^{m}\left|a_{i j}^{1}\right| L_{1 j}+\sum_{j=1}^{m}\left|a_{j i}^{2}\right| L_{2 i}+\sum_{j=1}^{m}\left|b_{i j}^{1}\right| L_{1 j} e^{\varepsilon \bar{\sigma}_{1 j}} \\
& +\sum_{j=1}^{m}\left|\alpha_{i j}^{1}\right| L_{1 j} \check{K}_{1 j}(\varepsilon)+\sum_{j=1}^{m}\left|\alpha_{j i}^{2}\right| L_{2 i} \check{K}_{2 i}(\varepsilon)+\sum_{j=1}^{m}\left|\beta_{i j}^{1}\right| L_{1 j} \check{K}_{1 j}(\varepsilon) \\
& +\sum_{j=1}^{m}\left|\beta_{j i}^{2}\right| L_{2 i} \check{K}_{2 i}(\varepsilon)+2 \mu_{1 i} e^{\varepsilon \tau_{1 i}}\left(\mu_{1 i} e^{\varepsilon \tau_{1 i}}-\varepsilon\right) \tau_{1 i}+\sum_{j=1}^{m} \frac{\left|b_{j i}^{2}\right| L_{2 i} e^{\varepsilon \bar{\sigma}_{2 i}} \bar{\sigma}_{2 i}}{1-\hat{\sigma}_{2 i}} \\
& +\sum_{j=1}^{m} \mu_{1 i} e^{\varepsilon \tau_{1 i} \mid}\left|a_{i j}^{1}\right| L_{1 j} \tau_{1 i}+\sum_{j=1}^{m} \mu_{2 j} e^{\varepsilon \tau_{2 j} j}\left|a_{j i}^{2}\right| L_{2 i} \tau_{2 j}+\sum_{j=1}^{m} \mu_{1 i} e^{\varepsilon \tau_{1 i} \mid}\left|b_{i j}^{1}\right| L_{1 j} e^{\varepsilon \bar{\sigma}_{1 j}} \tau_{1 i} \\
& +\sum_{j=1}^{m} \frac{\mu_{2 j} e^{\varepsilon \tau_{2 j}}\left|b_{j i}^{2}\right| L_{2 i} e^{\varepsilon \bar{\sigma}_{2 i}} \tau_{2 j} \bar{\sigma}_{2 i}}{1-\hat{\sigma}_{2 i}}+\sum_{j=1}^{m}\left|\alpha_{i j}^{1}\right| L_{1 j} \mu_{1 i} e^{\varepsilon \tau_{1 i} \check{K}_{1 j}(\varepsilon) \tau_{1 i}} \\
& +\sum_{j=1}^{m}\left|\alpha_{j i}^{2}\right| L_{2 i} \mu_{2 j} e^{\varepsilon \tau_{2 i}} \check{K}_{2 i}(\varepsilon) \tau_{2 j}+4 \sum_{i=1}^{n} \sum_{j=1}^{m}\left|\tilde{a}_{j i}^{2}\right|^{2}\left|\tilde{L}_{2 i}\right|^{2} \\
& +4 \sum_{i=1}^{n} \sum_{j=1}^{m} \frac{\left|\tilde{b}_{j i}^{2}\right|^{2}\left|\tilde{L}_{2 i}\right|^{2} \overline{\tilde{\sigma}}_{2 i}}{1-\hat{\tilde{\sigma}}_{2 i}}+4\left(\sum_{i=1}^{n} \sum_{j=1}^{m}\left|\tilde{\alpha}_{j i}^{2}\right|^{2}\left|\tilde{L}_{2 i}\right|^{2} \check{\tilde{K}}_{2 i}(\varepsilon)\right) \check{\tilde{K}}_{2 i}(\varepsilon) \\
& +4\left(\sum_{i=1}^{n} \sum_{j=1}^{m}\left|\tilde{\beta}_{j i}^{2}\right|^{2}\left|\tilde{L}_{2 i}\right|^{2} \check{\tilde{K}}_{2 i}(\varepsilon)\right) \check{\tilde{K}}_{2 i}(\varepsilon)
\end{aligned}
$$

and

$$
\begin{aligned}
& M_{2 j}(\varepsilon)=-2\left(\mu_{2 j} e^{\varepsilon \tau_{2 j}}-\varepsilon\right)+\sum_{i=1}^{n}\left|a_{i j}^{1}\right| L_{1 j}+\sum_{i=1}^{n}\left|a_{j i}^{2}\right| L_{2 i}+\sum_{i=1}^{n} \frac{\left|b_{i j}^{1}\right| L_{1 j} e^{\varepsilon \bar{\sigma}_{1 j}} \bar{\sigma}_{1 j}}{1-\hat{\sigma}_{1 j}} \\
& +\sum_{i=1}^{n}\left|\alpha_{i j}^{1}\right| L_{1 j} \check{K}_{1 j}(\varepsilon)+\sum_{i=1}^{n}\left|\alpha_{j i}^{2}\right| L_{2 i} \check{K}_{2 i}(\varepsilon)+\sum_{i=1}^{n}\left|\beta_{i j}^{1}\right| L_{1 j} \check{K}_{1 j}(\varepsilon) \\
& +\sum_{i=1}^{n}\left|\beta_{j i}^{2}\right| L_{2 i} \check{K}_{2 i}(\varepsilon)+2 \mu_{2 j} e^{\varepsilon \tau_{2 j}}\left(\mu_{2 j} e^{\varepsilon \tau_{2 j}}-\varepsilon\right) \tau_{2 j}+\sum_{i=1}^{n}\left|b_{j i}^{2}\right| L_{2 i} e^{\varepsilon \bar{\sigma}_{2 i}} \\
& +\sum_{i=1}^{n} \mu_{1 i} e^{\varepsilon \tau_{1 i}}\left|a_{i j}^{1}\right| L_{1 j} \tau_{1 i}+\sum_{i=1}^{n} \mu_{2 j} e^{\varepsilon \tau_{2 j}}\left|a_{j i}^{2}\right| L_{2 i} \tau_{2 j} \\
& +\sum_{i=1}^{n} \frac{\mu_{1 i} e^{\varepsilon \tau_{1 i}}\left|b_{i j}^{1}\right| L_{1 j} e^{\varepsilon \bar{\sigma}_{1 j}} \tau_{1 i} \bar{\sigma}_{1 j}}{1-\hat{\sigma}_{1 j}} \\
& +\sum_{i=1}^{n} \mu_{2 j} e^{\varepsilon \tau_{2 j}}\left|b_{j i}^{2}\right| L_{2 i} e^{\varepsilon \bar{\sigma}_{2 i}} \tau_{2 j}+\sum_{i=1}^{n}\left|\alpha_{i j}^{1}\right| L_{1 j} \mu_{1 i} e^{\varepsilon \tau_{1 i}} \breve{K}_{1 j}(\varepsilon) \tau_{1 i} \\
& +\sum_{i=1}^{n}\left|\alpha_{j i}^{2}\right| L_{2 i} \mu_{2 j} e^{\varepsilon \tau_{2 j}} \check{K}_{2 i}(\varepsilon) \tau_{2 j}+4 \sum_{i=1}^{n} \sum_{j=1}^{m}\left|\tilde{a}_{i j}^{1}\right|^{2}\left|\tilde{L}_{1 j}\right|^{2}
\end{aligned}
$$




$$
\begin{aligned}
& +4 \sum_{i=1}^{n} \sum_{j=1}^{m} \frac{\left|\tilde{b}_{i j}^{1}\right|^{2}\left|\tilde{L}_{1 j}\right|^{2} \overline{\tilde{\sigma}}_{1 j}}{1-\hat{\tilde{\sigma}}_{1 j}}+4\left(\sum_{i=1}^{n} \sum_{j=1}^{m}\left|\tilde{\alpha}_{j i}^{2}\right|^{2}\left|\tilde{L}_{2 i}\right|^{2} \check{\tilde{K}}_{1 j}(\varepsilon)\right) \check{\tilde{K}}_{1 j}(\varepsilon) \\
& +4\left(\sum_{i=1}^{n} \sum_{j=1}^{m}\left|\tilde{\beta}_{j i}^{2}\right|^{2}\left|\tilde{L}_{2 i}\right|^{2} \tilde{\tilde{K}}_{1 j}(\varepsilon)\right) \check{\tilde{K}}_{1 j}(\varepsilon) .
\end{aligned}
$$

Proof Let $\left(u_{1}^{*}, \ldots, u_{n}^{*}, v_{1}^{*}, \ldots, v_{m}^{*}\right)$ be the unique equilibrium state of system (2.1), and let $\left(u_{1}, \ldots, u_{n}, v_{1}, \ldots, v_{m}\right)$ be the solution to system (2.1) with initial data given by (2.2). Observe that $\left(\bar{u}_{1}(t), \ldots, \bar{u}_{n}(t), \bar{v}_{1}(t), \ldots, \bar{v}_{m}(t)\right)=\left(u_{1}(t)-u_{1}^{*}, \ldots, u_{n}(t)-u_{n}^{*}, v_{1}\left(v_{1}\right)-v_{1}^{*}, \ldots, v_{m}(t)-\right.$ $\left.v_{m}^{*}\right)$ is the unique solution to the following initial value problem

$$
\begin{aligned}
& \int d\left[\bar{u}_{i}(t)-\mu_{1 i} \int_{t-\tau_{1 i}}^{t} \bar{u}_{i}(\theta) d \theta\right] \\
& =\left[-\mu_{1 i} \bar{u}_{i}(t)+\sum_{j=1}^{m} a_{i j}^{1}\left(f_{1 j}\left(v_{j}^{*}+\bar{v}_{j}(t)\right)-f_{1 j}\left(v_{j}^{*}\right)\right)\right. \\
& +\sum_{j=1}^{m} b_{i j}^{1}\left(f_{1 j}\left(v_{j}^{*}+\bar{v}_{j}\left(t-\sigma_{1 j}(t)\right)\right)-f_{1 j}\left(v_{j}^{*}\right)\right) \\
& +\bigwedge_{j=1}^{m} \alpha_{i j}^{1} \int_{-\infty}^{t} K_{1 j}(t-s) f_{1 j}\left(v_{j}^{*}+\bar{v}_{j}(s)\right) d s \\
& -\bigwedge_{j=1}^{m} \alpha_{i j}^{1} \int_{-\infty}^{t} K_{1 j}(t-s) f_{1 j}\left(v_{j}^{*}\right) d s \\
& +\bigvee_{j=1}^{m} \beta_{i j}^{1} \int_{-\infty}^{t} K_{1 j}(t-s) f_{1 j}\left(v_{j}^{*}+\bar{v}_{j}(s)\right) d s \\
& \left.-\bigvee_{j=1}^{m} \beta_{i j}^{1} \int_{-\infty}^{t} K_{1 j}(t-s) f_{1 j}\left(v_{j}^{*}\right) d s\right] d t \\
& +\left[\sum_{j=1}^{m} \tilde{a}_{i j}^{1}\left(\tilde{f}_{1 j}\left(v_{j}^{*}+\bar{v}_{j}(t)\right)-\tilde{f}_{1 j}\left(v_{j}^{*}\right)\right)\right. \\
& +\sum_{j=1}^{m} \tilde{b}_{i j}^{1}\left(\tilde{f}_{1 j}\left(v_{j}^{*}+\bar{v}_{j}\left(t-\tilde{\sigma}_{1 j}(t)\right)\right)-\tilde{f}_{1 j}\left(v_{j}^{*}\right)\right) \\
& +\bigwedge_{j=1}^{m} \tilde{\alpha}_{i j}^{1} \int_{-\infty}^{t} \tilde{K}_{1 j}(t-s) \tilde{f}_{1 j}\left(v_{j}^{*}+\bar{v}_{j}(s)\right) d s \\
& -\bigwedge_{j=1}^{m} \tilde{\alpha}_{i j}^{1} \int_{-\infty}^{t} \tilde{K}_{1 j}(t-s) \tilde{f}_{1 j}\left(v_{j}^{*}\right) d s \\
& +\bigvee_{j=1}^{m} \tilde{\beta}_{i j}^{1} \int_{-\infty}^{t} \tilde{K}_{1 j}(t-s) \tilde{f}_{1 j}\left(v_{j}^{*}+\bar{v}_{j}(s)\right) d s \\
& \left.-\bigvee_{j=1}^{m} \tilde{\beta}_{i j}^{1} \int_{-\infty}^{t} \tilde{K}_{1 j}(t-s) \tilde{f}_{1 j}\left(v_{j}^{*}\right) d s\right] d B(t), \\
& d\left[\bar{v}_{j}(t)-\mu_{2 j} \int_{t-\tau_{2 j}}^{t} \bar{v}_{j}(\theta) d \theta\right] \\
& =\left[-\mu_{2 j} \bar{v}_{j}(t)+\sum_{i=1}^{n} a_{j i}^{2}\left(f_{2 i}\left(u_{i}^{*}+\bar{u}_{i}(t)\right)-f_{2 i}\left(u_{i}^{*}\right)\right)\right. \\
& +\sum_{i=1}^{n} b_{j i}^{2}\left(f_{2 i}\left(u_{i}^{*}+\bar{u}_{i}\left(t-\sigma_{2 i}(t)\right)\right)-f_{2 i}\left(u_{i}^{*}\right)\right) \\
& +\bigwedge_{i=1}^{n} \alpha_{j i}^{2} \int_{-\infty}^{t} K_{2 i}(t-s) f_{2 i}\left(u_{i}^{*}+\bar{u}_{i}(s)\right) d s \\
& -\bigwedge_{i=1}^{n} \alpha_{j i}^{2} \int_{-\infty}^{t} K_{2 i}(t-s) f_{2 i}\left(u_{i}^{*}\right) d s \\
& +\bigvee_{i=1}^{n} \beta_{j i}^{2} \int_{-\infty}^{t} K_{2 i}(t-s) f_{2 i}\left(u_{i}^{*}+\bar{u}_{i}(s)\right) d s \\
& \left.-\bigvee_{i=1}^{n} \beta_{j i}^{2} \int_{-\infty}^{t} K_{2 i}(t-s) f_{2 i}\left(u_{i}^{*}\right) d s\right] d t \\
& +\left[\sum_{i=1}^{n} \tilde{a}_{j i}^{2}\left(\tilde{f}_{2 i}\left(u_{i}^{*}+\bar{u}_{i}(t)\right)-\tilde{f}_{2 i}\left(u_{i}^{*}\right)\right)\right. \\
& +\sum_{i=1}^{n} \tilde{b}_{j i}^{2}\left(\tilde{f}_{2 i}\left(u_{i}^{*}+\bar{u}_{i}\left(t-\tilde{\sigma}_{2 i}(t)\right)\right)-\tilde{f}_{2 i}\left(u_{i}^{*}\right)\right) \\
& +\bigwedge_{i=1}^{n} \tilde{\alpha}_{j i}^{2} \int_{-\infty}^{t} \tilde{K}_{2 i}(t-s) \tilde{f}_{2 i}\left(u_{i}^{*}+\bar{u}_{i}(s)\right) d s \\
& -\bigwedge_{i=1}^{n} \tilde{\alpha}_{j i}^{2} \int_{-\infty}^{t} \tilde{K}_{2 i}(t-s) \tilde{f}_{2 i}\left(u_{i}^{*}\right) d s \\
& +\bigvee_{i=1}^{n} \tilde{\beta}_{j i}^{2} \int_{-\infty}^{t} \tilde{K}_{2 i}(t-s) \tilde{f}_{2 i}\left(u_{i}^{*}+\bar{u}_{i}(s)\right) d s \\
& \left.-\bigvee_{i=1}^{n} \tilde{\beta}_{j i}^{2} \int_{-\infty}^{t} \tilde{K}_{2 i}(t-s) \tilde{f}_{2 i}\left(u_{i}^{*}\right) d s\right] d B(t), \\
& \bar{u}_{i}(s)=\phi_{i}(s)-u_{i}^{*}, \quad \bar{v}_{j}(s)=\psi_{j}(s)-v_{j}^{*}, \quad \forall s \in(-\infty, 0], \mathbb{P} \text {-a.s., } \\
& i=1, \ldots, n, j=1, \ldots, m \text {. }
\end{aligned}
$$


Let

$$
U_{i}(t)= \begin{cases}\bar{u}_{i}(t), & t<0 \\ e^{\varepsilon t} \bar{u}_{i}(t), & t \geq 0\end{cases}
$$

for $i=1, \ldots, n$, and let

$$
V_{j}(t)= \begin{cases}\bar{v}_{j}(t), & t<0, \\ e^{\varepsilon t} \bar{v}_{j}(t), & t \geq 0,\end{cases}
$$

for $j=1, \ldots, m$. By Itô's rule, we have

$$
d \bar{u}_{i}(t)=e^{-\varepsilon t}\left(d U_{i}(t)-\varepsilon U_{i}(t) d t\right)
$$

and

$$
\begin{aligned}
d \int_{t-\tau_{1 i}}^{t} \bar{u}_{i}(s) d s & =d \int_{t-\tau_{1 i}}^{t} e^{-\varepsilon s} U_{i}(s) d s \\
& =\left[e^{-\varepsilon t} U_{i}(t)-e^{-\varepsilon\left(t-\tau_{1 i}\right)} U_{i}\left(t-\tau_{1 i}\right)\right] d t \\
& =e^{-\varepsilon t}\left[U_{i}(t)-e^{\varepsilon \tau_{1 i}} U_{i}\left(t-\tau_{1 i}\right)\right] d t \\
& =e^{-\varepsilon t}\left[\left(1-e^{\varepsilon \tau_{1 i}}\right) U_{i}(t) d t+e^{\varepsilon \tau_{1 i}} d \int_{t-\tau_{1 i}}^{t} U_{i}(s) d s\right] .
\end{aligned}
$$

Therefore

$$
\begin{aligned}
d[ & \left.\bar{u}_{i}(t)-\mu_{1 i} \int_{t-\tau_{1 i}}^{t} \bar{u}_{i}(\theta) d \theta\right]+\mu_{1 i} \bar{u}_{i}(t) d t \\
= & d \bar{u}_{i}(t)-\mu_{1 i} d \int_{t-\tau_{1 i}}^{t} \bar{u}_{i}(\theta) d \theta+\mu_{1 i} \bar{u}_{i}(t) d t \\
= & e^{-\varepsilon t}\left(d U_{i}(t)-\varepsilon U_{i}(t) d t\right)+\mu_{1 i} e^{-\varepsilon t} U_{i}(t) d t \\
& -\mu_{1 i} e^{-\varepsilon t}\left[\left(1-e^{\varepsilon \tau_{1 i}}\right) U_{i}(t) d t+e^{\varepsilon \tau_{1 i}} d \int_{t-\tau_{1 i}}^{t} U_{i}(s) d s\right] \\
= & e^{-\varepsilon t}\left\{d\left[U_{i}(t)-\mu_{1 i} e^{\varepsilon \tau_{1 i}} \int_{t-\tau_{1 i}}^{t} U_{i}(s) d s\right]+\left(\mu_{1 i} e^{\varepsilon \tau_{1 i}}-\varepsilon\right) U_{i}(t) d t\right\}, \quad i=1, \ldots, n .
\end{aligned}
$$

Similarly, we have

$$
\begin{aligned}
d[ & \left.\bar{v}_{j}(t)-\mu_{2 j} \int_{t-\tau_{2 j}}^{t} \bar{v}_{j}(\theta) d \theta\right]+\mu_{2 j} \bar{v}_{j}(t) d t \\
& =e^{-\varepsilon t}\left\{d\left[V_{j}(t)-\mu_{2 j} e^{\varepsilon \tau_{2 j} j} \int_{t-\tau_{2 j}}^{t} V_{j}(s) d s\right]+\left(\mu_{2 j} e^{\varepsilon \tau_{2 j}}-\varepsilon\right) V_{j}(t) d t\right\}, \quad j=1, \ldots, m .
\end{aligned}
$$

With these preparations in hand, we can deduce that, for every solution $\left(\bar{u}_{1}, \ldots, \bar{u}_{n}, \bar{v}_{1}, \ldots\right.$, $\left.\bar{v}_{m}\right)$ to IVP (3.2), $\left(U_{1}, \ldots, U_{n}, V_{1}, \ldots, V_{m}\right)$ is the unique solution to the initial value prob- 
lem

$$
\begin{aligned}
& \int d\left[U_{i}(t)-\mu_{1 i} e^{\varepsilon \tau_{1 i}} \int_{t-\tau_{1 i}}^{t} U_{i}(s) d s\right] \\
& =\left[-\left(\mu_{1 i} e^{\varepsilon \tau_{1 i}}-\varepsilon\right) U_{i}(t)+\sum_{j=1}^{m} a_{i j}^{1} e^{\varepsilon t}\left(f_{1 j}\left(v_{j}^{*}+e^{-\varepsilon t} V_{j}(t)\right)-f_{1 j}\left(v_{j}^{*}\right)\right)\right. \\
& +\sum_{j=1}^{m} b_{i j}^{1} e^{\varepsilon t}\left(f_{1 j}\left(v_{j}^{*}+e^{-\varepsilon\left(t-\sigma_{1 j}(t)\right)} V_{j}\left(t-\sigma_{1 j}(t)\right)\right)-f_{1 j}\left(v_{j}^{*}\right)\right) \\
& +\bigwedge_{j=1}^{m} \alpha_{i j}^{1} e^{\varepsilon t} \int_{-\infty}^{t} K_{1 j}(t-s) f_{1 j}\left(v_{j}^{*}+e^{-\varepsilon s} V_{j}(s)\right) d s \\
& -\bigwedge_{j=1}^{m} \alpha_{i j}^{1} e^{\varepsilon t} \int_{-\infty}^{t} K_{1 j}(t-s) f_{1 j}\left(v_{j}^{*}\right) d s \\
& +\bigvee_{j=1}^{m} \beta_{i j}^{1} e^{\varepsilon t} \int_{-\infty}^{t} K_{1 j}(t-s) f_{1 j}\left(v_{j}^{*}+e^{-\varepsilon s} V_{j}(s)\right) d s \\
& \left.-\bigvee_{j=1}^{m} \beta_{i j}^{1} e^{\varepsilon t} \int_{-\infty}^{t} K_{1 j}(t-s) f_{1 j}\left(v_{j}^{*}\right) d s\right] d t \\
& +\left[\sum_{j=1}^{m} \tilde{a}_{i j}^{1} e^{\varepsilon t}\left(\tilde{f}_{1 j}\left(v_{j}^{*}+e^{-\varepsilon t} V_{j}(t)\right)-\tilde{f}_{1 j}\left(v_{j}^{*}\right)\right)\right. \\
& +\sum_{j=1}^{m} \tilde{b}_{i j}^{1} e^{\varepsilon t}\left(\tilde{f}_{1 j}\left(v_{j}^{*}+e^{-\varepsilon\left(t-\tilde{\sigma}_{1 j}(t)\right)} V_{j}\left(t-\tilde{\sigma}_{1 j}(t)\right)\right)-\tilde{f}_{1 j}\left(v_{j}^{*}\right)\right) \\
& +\bigwedge_{j=1}^{m} \tilde{\alpha}_{i j}^{1} e^{\varepsilon t} \int_{-\infty}^{t} \tilde{K}_{1 j}(t-s) \tilde{f}_{1 j}\left(v_{j}^{*}+e^{-\varepsilon s} V_{j}(s)\right) d s \\
& -\bigwedge_{j=1}^{m} \tilde{\alpha}_{i j}^{1} e^{\varepsilon t} \int_{-\infty}^{t} \tilde{K}_{1 j}(t-s) \tilde{f}_{1 j}\left(v_{j}^{*}\right) d s \\
& +\bigvee_{j=1}^{m} \tilde{\beta}_{i j}^{1} e^{\varepsilon t} \int_{-\infty}^{t} \tilde{K}_{1 j}(t-s) \tilde{f}_{1 j}\left(v_{j}^{*}+e^{-\varepsilon s} V_{j}(s)\right) d s \\
& \left.-\bigvee_{j=1}^{m} \tilde{\beta}_{i j}^{1} e^{\varepsilon t} \int_{-\infty}^{t} \tilde{K}_{1 j}(t-s) \tilde{f}_{1 j}\left(v_{j}^{*}\right) d s\right] d B(t) \\
& d\left[V_{j}(t)-\mu_{2 j} e^{\varepsilon \tau_{2 j}} \int_{t-\tau_{2 j}}^{t} V_{j}(s) d s\right] \\
& =\left[-\left(\mu_{2 j} e^{\varepsilon \tau_{2 j}}-\varepsilon\right) V_{j}(t)+\sum_{i=1}^{n} a_{j i}^{2} e^{\varepsilon t}\left(f_{2 i}\left(u_{i}^{*}+e^{-\varepsilon t} U_{i}(t)\right)-f_{2 i}\left(u_{i}^{*}\right)\right)\right. \\
& +\sum_{i=1}^{n} b_{j i}^{2} e^{\varepsilon t}\left(f_{2 i}\left(u_{i}^{*}+e^{-\varepsilon\left(t-\sigma_{2 i}(t)\right)} U_{i}\left(t-\sigma_{2 i}(t)\right)\right)-f_{2 i}\left(u_{i}^{*}\right)\right) \\
& +\bigwedge_{i=1}^{n} \alpha_{j i}^{2} e^{\varepsilon t} \int_{-\infty}^{t} K_{2 i}(t-s) f_{2 i}\left(u_{i}^{*}+e^{-\varepsilon s} U_{i}(s)\right) d s \\
& -\bigwedge_{i=1}^{n} \alpha_{j i}^{2} e^{\varepsilon t} \int_{-\infty}^{t} K_{2 i}(t-s) f_{2 i}\left(u_{i}^{*}\right) d s \\
& +\bigvee_{i=1}^{n} \beta_{j i}^{2} e^{\varepsilon t} \int_{-\infty}^{t} K_{2 i}(t-s) f_{2 i}\left(u_{i}^{*}+e^{-\varepsilon s} U_{i}(s)\right) d s \\
& \left.-\bigvee_{i=1}^{n} \beta_{j i}^{2} e^{\varepsilon t} \int_{-\infty}^{t} K_{2 i}(t-s) f_{2 i}\left(u_{i}^{*}\right) d s\right] d t \\
& +\left[\sum_{i=1}^{n} \tilde{a}_{j i}^{2} e^{\varepsilon t}\left(\tilde{f}_{2 i}\left(u_{i}^{*}+e^{-\varepsilon t} U_{i}(t)\right)-\tilde{f}_{2 i}\left(u_{i}^{*}\right)\right)\right. \\
& +\sum_{i=1}^{n} \tilde{b}_{j i}^{2} e^{\varepsilon t}\left(\tilde{f}_{2 i}\left(u_{i}^{*}+e^{-\varepsilon\left(t-\tilde{\sigma}_{2 i}(t)\right)} U_{i}\left(t-\tilde{\sigma}_{2 i}(t)\right)\right)-\tilde{f}_{2 i}\left(u_{i}^{*}\right)\right) \\
& +\bigwedge_{i=1}^{n} \tilde{\alpha}_{j i}^{2} e^{\varepsilon t} \int_{-\infty}^{t} \tilde{K}_{2 i}(t-s) \tilde{f}_{2 i}\left(u_{i}^{*}+e^{-\varepsilon s} U_{i}(s)\right) d s \\
& -\bigwedge_{i=1}^{n} \tilde{\alpha}_{j i}^{2} e^{\varepsilon t} \int_{-\infty}^{t} \tilde{K}_{2 i}(t-s) \tilde{f}_{2 i}\left(u_{i}^{*}\right) d s \\
& +\bigvee_{i=1}^{n} \tilde{\beta}_{j i}^{2} e^{\varepsilon t} \int_{-\infty}^{t} \tilde{K}_{2 i}(t-s) \tilde{f}_{2 i}\left(u_{i}^{*}+e^{-\varepsilon s} U_{i}(s)\right) d s \\
& \left.-\bigvee_{i=1}^{n} \tilde{\beta}_{j i}^{2} e^{\varepsilon t} \int_{-\infty}^{t} \tilde{K}_{2 i}(t-s) \tilde{f}_{2 i}\left(u_{i}^{*}\right) d s\right] d B(t), \\
& U_{i}(s)=\phi_{i}(s)-u_{i}^{*}, \quad V_{j}(s)=\psi_{j}(s)-v_{j}^{*}, \quad \forall s \in(-\infty, 0], \mathbb{P} \text {-a.s., } \\
& i=1, \ldots, n, j=1, \ldots, m \text {. }
\end{aligned}
$$

Let $\mathcal{V}(t ; \varepsilon)=\mathbb{E} \sum_{k=1}^{4} \mathcal{V}_{k}(t ; \varepsilon)$, where $\mathcal{V}_{k}(t ; \varepsilon)(k=1,2,3,4)$ are defined by

$$
\begin{aligned}
\mathcal{V}_{1}(t ; \varepsilon)= & \sum_{i=1}^{n}\left|U_{i}(t)-\mu_{1 i} e^{\varepsilon \tau_{1 i}} \int_{t-\tau_{1 i}}^{t} U_{i}(s) d s\right|^{2} \\
\mathcal{V}_{2}(t ; \varepsilon)= & \sum_{i=1}^{n} \sum_{j=1}^{m} \frac{\left|b_{j i}^{2}\right| L_{2 i} e^{\varepsilon \bar{\sigma}_{2 i}}}{1-\hat{\sigma}_{2 i}} \int_{t-\sigma_{2 i}(t)}^{t}\left|U_{i}(s)\right|^{2} d s \\
& +\sum_{i=1}^{n} \sum_{j=1}^{m}\left|\alpha_{j i}^{2}\right| L_{2 i} \int_{0}^{+\infty} e^{\varepsilon s} K_{2 i}(s) \int_{t-s}^{t}\left|U_{i}(\theta)\right|^{2} d \theta d s
\end{aligned}
$$




$$
\begin{aligned}
& +\sum_{i=1}^{n} \sum_{j=1}^{m}\left|\beta_{j i}^{2}\right| L_{2 i} \int_{0}^{+\infty} e^{\varepsilon s} K_{2 i}(s) \int_{t-s}^{t}\left|U_{i}(\theta)\right|^{2} d \theta d s \\
& +\sum_{i=1}^{n} \mu_{1 i} e^{\varepsilon \tau_{1 i}}\left(\mu_{1 i} e^{\varepsilon \tau_{1 i}}-\varepsilon\right) \int_{t-\tau_{1 i}}^{t} \int_{\tau}^{t}\left|U_{i}(s)\right|^{2} d s d \tau \\
& +\sum_{i=1}^{n} \sum_{j=1}^{m} \mu_{1 i} e^{\varepsilon \tau_{1 i}}\left|a_{i j}^{1}\right| L_{1 j} \int_{t-\tau_{1 i}}^{t} \int_{\tau}^{t}\left|U_{i}(s)\right|^{2} d s d \tau \\
& +\sum_{i=1}^{n} \sum_{j=1}^{m} \mu_{1 i} e^{\varepsilon \tau_{1 i}}\left|b_{i j}^{1}\right| L_{1 j} e^{\varepsilon \bar{\sigma}_{1 j}} \int_{t-\tau_{1 i}}^{t} \int_{\tau}^{t}\left|U_{i}(s)\right|^{2} d s d \tau \\
& +\sum_{i=1}^{n} \sum_{j=1}^{m} \frac{\mu_{2 j} e^{\varepsilon \tau_{2 j}}\left|b_{j i}^{2}\right| L_{2 i} e^{\varepsilon \bar{\sigma}_{2 i}} \tau_{2 j}}{1-\hat{\sigma}_{2 i}} \int_{t-\sigma_{2 i}(t)}^{t}\left|U_{i}(s)\right|^{2} d s \\
& +\sum_{i=1}^{n} \sum_{j=1}^{m}\left|\alpha_{i j}^{1}\right| L_{1 j} \mu_{1 i} e^{\varepsilon \tau \tau_{1 i}} \check{K}_{1 j}(\varepsilon) \int_{t-\tau_{1 i}}^{t} \int_{\tau}^{t}\left|U_{i}(s)\right|^{2} d s d \tau \\
& +\sum_{i=1}^{n} \sum_{j=1}^{m}\left|\alpha_{j i}^{2}\right| L_{2 i} \mu_{2 j} e^{\varepsilon \tau_{2 j}} \tau_{2 j} \int_{0}^{+\infty} e^{\varepsilon s} K_{2 i}(s) \int_{t-s}^{t}\left|U_{i}(\theta)\right|^{2} d \theta d s
\end{aligned}
$$

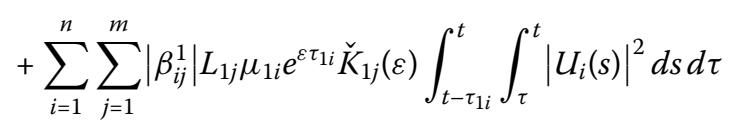

$$
\begin{aligned}
& +\sum_{i=1}^{n} \sum_{j=1}^{m}\left|\beta_{j i}^{2}\right| L_{2 i} \mu_{2 j} e^{\varepsilon \tau_{2 j}} \tau_{2 j} \int_{0}^{+\infty} e^{\varepsilon s} K_{2 i}(s) \int_{t-s}^{t}\left|U_{i}(\theta)\right|^{2} d \theta d s \\
& +4\left(\sum_{i=1}^{n} \sum_{j=1}^{m} \frac{\left|\tilde{b}_{j i}^{2}\right|^{2}\left|\tilde{L}_{2 i}\right|^{2}}{1-\hat{\tilde{\sigma}}_{2 i}}\right) \sum_{i=1}^{n} \int_{t-\tilde{\sigma}_{2 i}(t)}^{t}\left|U_{i}(s)\right|^{2} d s \\
& +4\left(\sum_{i=1}^{n} \sum_{j=1}^{m}\left|\tilde{\alpha}_{j i}^{2}\right|^{2}\left|\tilde{L}_{2 i}\right|^{2} \check{\tilde{K}}_{2 i}(\varepsilon)\right) \sum_{i=1}^{n} \int_{0}^{+\infty} \tilde{K}_{2 i}(s) e^{\varepsilon s} \int_{t-s}^{t}\left|U_{i}(\theta)\right|^{2} d \theta d s \\
& +4\left(\sum_{i=1}^{n} \sum_{j=1}^{m}\left|\tilde{\beta}_{j i}^{2}\right|^{2}\left|\tilde{L}_{2 i}\right|^{2} \tilde{\tilde{K}}_{2 i}(\varepsilon)\right) \sum_{i=1}^{n} \int_{0}^{+\infty} \tilde{K}_{2 i}(s) e^{\varepsilon s} \int_{t-s}^{t}\left|U_{i}(\theta)\right|^{2} d \theta d s, \\
& \mathcal{V}_{3}(t ; \varepsilon)=\sum_{j=1}^{m}\left|V_{j}(t)-\mu_{2 j} e^{\varepsilon \tau_{2 j}} \int_{t-\tau_{2 j}}^{t} V_{j}(s) d s\right|^{2},
\end{aligned}
$$

and

$$
\begin{aligned}
\mathcal{V}_{4}(t ; \varepsilon)= & \sum_{j=1}^{m} \sum_{i=1}^{n} \frac{\left|b_{i j}^{1}\right| L_{1 j} e^{\varepsilon \bar{\sigma}_{1 j}}}{1-\hat{\sigma}_{1 j}} \int_{t-\sigma_{1 j}(t)}^{t}\left|V_{j}(s)\right|^{2} d s \\
& +\sum_{j=1}^{m} \sum_{i=1}^{n}\left|\alpha_{i j}^{1}\right| L_{1 j} \int_{0}^{+\infty} e^{\varepsilon s} K_{1 j}(s) \int_{t-s}^{t}\left|V_{j}(\theta)\right|^{2} d \theta d s \\
& +\sum_{j=1}^{m} \sum_{i=1}^{n}\left|\beta_{i j}^{1}\right| L_{1 j} \int_{0}^{+\infty} e^{\varepsilon s} K_{1 j}(s) \int_{t-s}^{t}\left|V_{j}(\theta)\right|^{2} d \theta d s
\end{aligned}
$$




$$
\begin{aligned}
& +\sum_{j=1}^{m} \mu_{2 j} e^{\varepsilon \tau_{2 j}}\left(\mu_{2 j} e^{\varepsilon \tau_{2 j}}-\varepsilon\right) \int_{t-\tau_{2 j}}^{t} \int_{\tau}^{t}\left|V_{j}(s)\right|^{2} d s d \tau \\
& +\sum_{j=1}^{m} \sum_{i=1}^{n} \mu_{2 j} e^{\varepsilon \tau_{2 j}}\left|a_{j i}^{2}\right| L_{2 i} \int_{t-\tau_{2 j}}^{t} \int_{\tau}^{t}\left|V_{j}(s)\right|^{2} d s d \tau \\
& +\sum_{j=1}^{m} \sum_{i=1}^{n} \frac{\mu_{1 i} e^{\varepsilon \tau_{1 i}}\left|b_{i j}^{1}\right| L_{1 j} e^{\varepsilon \bar{\sigma}_{1 j}} \tau_{1 i}}{1-\hat{\sigma}_{1 j}} \int_{t-\sigma_{1 j}(t)}^{t}\left|V_{j}(s)\right|^{2} d s \\
& +\sum_{j=1}^{m} \sum_{i=1}^{n} \mu_{2 j} e^{\varepsilon \tau_{2 j}}\left|b_{j i}^{2}\right| L_{2 i} e^{\varepsilon \bar{\varepsilon}_{2 i}} \int_{t-\tau_{2 j}}^{t} \int_{\tau}^{t}\left|V_{j}(s)\right|^{2} d s d \tau \\
& +\sum_{j=1}^{m} \sum_{i=1}^{n}\left|\alpha_{i j}^{1}\right| L_{1 j} \mu_{1 i} e^{\varepsilon \tau_{1 i}} \tau_{1 i} \int_{0}^{+\infty} e^{\varepsilon s} K_{1 j}(s) \int_{t-s}^{t}\left|V_{j}(\theta)\right|^{2} d \theta d s \\
& +\sum_{j=1}^{m} \sum_{i=1}^{n}\left|\alpha_{j i}^{2}\right| L_{2 i} \mu_{2 j} e^{\varepsilon \tau_{2 j}} \check{K}_{2 i}(\varepsilon) \int_{t-\tau_{2 j}}^{t} \int_{\tau}^{t}\left|V_{j}(s)\right|^{2} d s d \tau \\
& +\sum_{j=1}^{m} \sum_{i=1}^{n}\left|\beta_{i j}^{1}\right| L_{1 j} \mu_{1 i} e^{\varepsilon \tau_{1 i}} \tau_{1 i} \int_{0}^{+\infty} e^{\varepsilon s} K_{1 j}(s) \int_{t-s}^{t}\left|V_{j}(\theta)\right|^{2} d \theta d s \\
& +\sum_{j=1}^{m} \sum_{i=1}^{n}\left|\beta_{j i}^{2}\right| L_{2 i} \mu_{2 j} e^{\varepsilon \tau_{2 j}} \check{K}_{2 i}(\varepsilon) \int_{t-\tau_{2 j}}^{t} \int_{\tau}^{t}\left|V_{j}(s)\right|^{2} d s d \tau \\
& +4\left(\sum_{j=1}^{m} \sum_{i=1}^{n} \frac{\left|\tilde{b}_{i j}^{1}\right|^{2}\left|\tilde{L}_{1 j}\right|^{2}}{1-\hat{\tilde{\sigma}}_{1 j}}\right) \sum_{j=1}^{m} \int_{t-\tilde{\sigma}_{1 j}(t)}^{t}\left|V_{j}(s)\right|^{2} d s \\
& +4\left(\sum_{i=1}^{n} \sum_{j=1}^{m}\left|\tilde{\alpha}_{i j}^{1}\right|^{2}\left|\tilde{L}_{1 j}\right|^{2} \check{\tilde{K}}_{1 j}(\varepsilon)\right) \sum_{j=1}^{m} \int_{0}^{+\infty} \tilde{K}_{1 j}(s) e^{\varepsilon s} \int_{t-s}^{t}\left|V_{j}(\theta)\right|^{2} d \theta d s \\
& +4\left(\sum_{i=1}^{n} \sum_{j=1}^{m}\left|\tilde{\beta}_{1 j}^{1}\right|^{2}\left|\tilde{L}_{1 j}\right|^{2} \check{\tilde{K}}_{1 j}(\varepsilon)\right) \sum_{j=1}^{m} \int_{0}^{+\infty} \tilde{K}_{1 j}(s) e^{\varepsilon s} \int_{t-s}^{t}\left|V_{j}(\theta)\right|^{2} d \theta d s
\end{aligned}
$$

Let us denote

$$
\left\{\begin{array}{l}
d\left[U_{i}(t)-\mu_{1 i} e^{\varepsilon \tau_{1 i}} \int_{t-\tau_{1 i}}^{t} U_{i}(s) d s\right]=\mathfrak{a}_{1 i}(t) d t+\mathfrak{a}_{2 i}(t) d B(t), \quad i=1, \ldots, n, \\
d\left[V_{j}(t)-\mu_{2 j} e^{\varepsilon \tau_{2 j}} \int_{t-\tau_{2 j}}^{t} V_{j}(s) d s\right]=\mathfrak{b}_{1 j}(t) d t+\mathfrak{b}_{2 j}(t) d B(t), \quad j=1, \ldots, m
\end{array}\right.
$$

By Itô's formula,

$$
\begin{aligned}
d \mathcal{V}_{1}(t ; \varepsilon)= & d \sum_{i=1}^{n}\left|U_{i}(t)-\mu_{1 i} e^{\varepsilon \tau_{1 i}} \int_{t-\tau_{1 i}}^{t} U_{i}(s) d s\right|^{2} \\
= & \sum_{i=1}^{n}\left\{2 \mathfrak{a}_{1 i}(t)\left[U_{i}(t)-\mu_{1 i} e^{\varepsilon \tau_{1 i}} \int_{t-\tau_{1 i}}^{t} U_{i}(s) d s\right]+\left|\mathfrak{a}_{2 i}(t)\right|^{2}\right\} d t \\
& +2 \sum_{i=1}^{n}\left[U_{i}(t)-\mu_{1 i} e^{\varepsilon \tau_{1 i}} \int_{t-\tau_{1 i}}^{t} U_{i}(s) d s\right] \mathfrak{a}_{2 i}(t) d B(t) .
\end{aligned}
$$


Thanks to (3.3) and (3.4), we have

$$
\begin{aligned}
& \sum_{i=1}^{n} \mathfrak{a}_{1 i}(t)\left[U_{i}(t)-\mu_{1 i} e^{\varepsilon \tau_{1 i}} \int_{t-\tau_{1 i}}^{t} U_{i}(s) d s\right] \\
& =-\sum_{i=1}^{n}\left(\mu_{1 i} e^{\varepsilon \tau_{1 i}}-\varepsilon\right)\left|U_{i}(t)\right|^{2} \\
& +\sum_{i=1}^{n} \sum_{j=1}^{m} a_{i j}^{1} e^{\varepsilon t} U_{i}(t)\left(f_{1 j}\left(v_{j}^{*}+e^{-\varepsilon t} V_{j}(t)\right)-f_{1 j}\left(v_{j}^{*}\right)\right) \\
& +\sum_{i=1}^{n} \sum_{j=1}^{m} b_{i j}^{1} e^{\varepsilon t} U_{i}(t)\left(f_{1 j}\left(v_{j}^{*}+e^{-\varepsilon\left(t-\sigma_{1 j}(t)\right)} V_{j}\left(t-\sigma_{1 j}(t)\right)\right)-f_{1 j}\left(v_{j}^{*}\right)\right) \\
& +\sum_{i=1}^{n} e^{\varepsilon t} U_{i}(t)\left[\bigwedge_{j=1}^{m} \alpha_{i j}^{1} \int_{-\infty}^{t} K_{1 j}(t-s) f_{1 j}\left(v_{j}^{*}+e^{-\varepsilon s} V_{j}(s)\right) d s\right. \\
& \left.-\bigwedge_{j=1}^{m} \alpha_{i j}^{1} \int_{-\infty}^{t} K_{1 j}(t-s) f_{1 j}\left(v_{j}^{*}\right) d s\right] \\
& +\sum_{i=1}^{n} e^{\varepsilon t} U_{i}(t)\left[\bigvee_{j=1}^{m} \beta_{i j}^{1} \int_{-\infty}^{t} K_{1 j}(t-s) f_{1 j}\left(v_{j}^{*}+e^{-\varepsilon s} V_{j}(s)\right) d s\right. \\
& \left.-\bigvee_{j=1}^{m} \beta_{i j}^{1} \int_{-\infty}^{t} K_{1 j}(t-s) f_{1 j}\left(v_{j}^{*}\right) d s\right] \\
& +\sum_{i=1}^{n} \mu_{1 i} e^{\varepsilon \tau_{1 i}}\left(\mu_{1 i} e^{\varepsilon \tau_{1 i}}-\varepsilon\right) U_{i}(t) \int_{t-\tau_{1 i}}^{t} U_{i}(s) d s \\
& -\sum_{i=1}^{n} \sum_{j=1}^{m} \mu_{1 i} e^{\varepsilon \tau_{1 i}} a_{i j}^{1} e^{\varepsilon t} \int_{t-\tau_{1 i}}^{t} U_{i}(s) d s\left(f_{1 j}\left(v_{j}^{*}+e^{-\varepsilon t} V_{j}(t)\right)-f_{1 j}\left(v_{j}^{*}\right)\right) \\
& -\sum_{i=1}^{n} \sum_{j=1}^{m} \mu_{1 i} e^{\varepsilon \tau_{1 i}} b_{i j}^{1} e^{\varepsilon t} \\
& \times \int_{t-\tau_{1 i}}^{t} U_{i}(s) d s\left(f_{1 j}\left(v_{j}^{*}+e^{-\varepsilon\left(t-\sigma_{1 j}(t)\right)} V_{j}\left(t-\sigma_{1 j}(t)\right)\right)-f_{1 j}\left(v_{j}^{*}\right)\right) \\
& -\sum_{i=1}^{n} \mu_{1 i} e^{\varepsilon \tau_{1 i}} e^{\varepsilon t} \int_{t-\tau_{1 i}}^{t} U_{i}(s) d s \\
& \times\left[\bigwedge_{j=1}^{m} \alpha_{i j}^{1} \int_{-\infty}^{t} K_{1 j}(t-s) f_{1 j}\left(v_{j}^{*}+e^{-\varepsilon s} V_{j}(s)\right) d s-\bigwedge_{j=1}^{m} \alpha_{i j}^{1} \int_{-\infty}^{t} K_{1 j}(t-s) f_{1 j}\left(v_{j}^{*}\right) d s\right] \\
& -\sum_{i=1}^{n} \mu_{1 i} e^{\varepsilon \tau_{1 i}} e^{\varepsilon t} \int_{t-\tau_{1 i}}^{t} U_{i}(s) d s \\
& \times\left[\bigvee_{j=1}^{m} \beta_{i j}^{1} \int_{-\infty}^{t} K_{1 j}(t-s) f_{1 j}\left(v_{j}^{*}+e^{-\varepsilon s} V_{j}(s)\right) d s\right. \\
& \left.-\bigvee_{j=1}^{m} \beta_{i j}^{1} \int_{-\infty}^{t} K_{1 j}(t-s) f_{1 j}\left(v_{j}^{*}\right) d s\right]
\end{aligned}
$$


Let us spare some space to analyze the right-hand side of (3.6) term-by-term. Firstly, we have

$$
\begin{gathered}
2\left|\sum_{i=1}^{n} \sum_{j=1}^{m} a_{i j}^{1} e^{\varepsilon t} U_{i}(t)\left(f_{1 j}\left(v_{j}^{*}+e^{-\varepsilon t} V_{j}(t)\right)-f_{1 j}\left(v_{j}^{*}\right)\right)\right| \\
\leq 2 \sum_{i=1}^{n} \sum_{j=1}^{m}\left|a_{i j}^{1}\right| e^{\varepsilon t}\left|U_{i}(t)\right|\left|f_{1 j}\left(v_{j}^{*}+e^{-\varepsilon t} V_{j}(t)\right)-f_{1 j}\left(v_{j}^{*}\right)\right| \\
\leq \sum_{i=1}^{n} \sum_{j=1}^{m}\left|L_{1 j} a_{i j}^{1}\right|\left|U_{i}(t)\right|^{2}+\sum_{j=1}^{m} \sum_{i=1}^{n}\left|L_{1 j} a_{i j}^{1}\right|\left|V_{j}(t)\right|^{2},
\end{gathered}
$$

where the first inequality follows from the Cauchy-Schwarz inequality. We have

$$
\begin{aligned}
2 \mid & \sum_{i=1}^{n} \sum_{j=1}^{m} b_{i j}^{1} e^{\varepsilon t} U_{i}(t)\left(f_{1 j}\left(v_{j}^{*}+e^{-\varepsilon\left(t-\sigma_{1 j}(t)\right)} V_{j}\left(t-\sigma_{1 j}(t)\right)\right)-f_{1 j}\left(v_{j}^{*}\right)\right) \mid \\
\leq & 2 \sum_{i=1}^{n} \sum_{j=1}^{m}\left|L_{1 j} b_{i j}^{1}\right| e^{\varepsilon \sigma_{1 j}(t)}\left|U_{i}(t)\right|\left|V_{j}\left(t-\sigma_{1 j}(t)\right)\right| \\
\leq & \sum_{i=1}^{n} \sum_{j=1}^{m}\left|L_{1 j} b_{i j}^{1}\right| e^{\varepsilon \bar{\sigma}_{1 j}}\left|U_{i}(t)\right|^{2}+\sum_{j=1}^{m} \sum_{i=1}^{n}\left|L_{1 j} b_{i j}^{1}\right| e^{\varepsilon \bar{\sigma}_{1 j}}\left|V_{j}\left(t-\sigma_{1 j}(t)\right)\right|^{2} \\
= & \sum_{i=1}^{n} \sum_{j=1}^{m}\left|L_{1 j} b_{i j}^{1}\right| e^{\varepsilon \bar{\sigma}_{1 j}}\left|U_{i}(t)\right|^{2}-\sum_{j=1}^{m} \sum_{i=1}^{n} \frac{\left|L_{1 j} b_{i j}^{1}\right| e^{\varepsilon \bar{\sigma}_{1 j}}}{1-\hat{\sigma}_{1 j}} \frac{d}{d t} \int_{t-\sigma_{1 j}(t)}^{t}\left|V_{j}(s)\right|^{2} d s \\
& +\sum_{j=1}^{m} \sum_{i=1}^{n} \frac{\left|b_{i j}^{1}\right| L_{1 j} e^{\varepsilon \bar{\sigma}_{1 j}}\left(\dot{\sigma}_{1 j}(t)-\hat{\sigma}_{1 j}\right)}{1-\hat{\sigma}_{1 j}}\left|V_{j}\left(t-\sigma_{1 j}(t)\right)\right|^{2} d t \\
& +\sum_{j=1}^{m} \sum_{i=1}^{n} \frac{\left|L_{1 j} b_{i j}^{1}\right| e^{\varepsilon \bar{\sigma}_{1 j}} \bar{\sigma}_{1 j}}{1-\hat{\sigma}_{1 j}}\left|V_{j}(t)\right|^{2},
\end{aligned}
$$

where the first inequality follows from the triangle inequality and the definition of $L_{1 j}$, the second inequality follows from the Cauchy-Schwarz inequality, and the equality follows from some routine calculations. Further, we have

$$
\begin{aligned}
& 2 \mid \sum_{i=1}^{n} e^{\varepsilon t} U_{i}(t)\left[\bigwedge_{j=1}^{m} \alpha_{i j}^{1} \int_{-\infty}^{t} K_{1 j}(t-s) f_{1 j}\left(v_{j}^{*}+e^{-\varepsilon s} V_{j}(s)\right) d s\right. \\
& \left.\quad-\bigwedge_{j=1}^{m} \alpha_{i j}^{1} \int_{-\infty}^{t} K_{1 j}(t-s) f_{1 j}\left(v_{j}^{*}\right) d s\right] \mid \\
& \leq 2 \sum_{i=1}^{n} e^{\varepsilon t}\left|U_{i}(t)\right| \bigwedge_{j=1}^{m} \alpha_{i j}^{1} \int_{-\infty}^{t} K_{1 j}(t-s) f_{1 j}\left(v_{j}^{*}+e^{-\varepsilon s} V_{j}(s)\right) d s \\
& \quad-\bigwedge_{j=1}^{m} \alpha_{i j}^{1} \int_{-\infty}^{t} K_{1 j}(t-s) f_{1 j}\left(v_{j}^{*}\right) d s \mid \\
& \leq 2 \sum_{i=1}^{n} \sum_{j=1}^{m} L_{1 j}\left|U_{i}(t)\right|\left|\alpha_{i j}^{1}\right| \int_{-\infty}^{t} e^{\varepsilon(t-s)} K_{1 j}(t-s)\left|V_{j}(s)\right| d s
\end{aligned}
$$




$$
\begin{aligned}
\leq & \sum_{i=1}^{n} \sum_{j=1}^{m}\left|L_{1 j} \alpha_{i j}^{1}\right| \check{K}_{1 j}(\varepsilon)\left|U_{i}(t)\right|^{2}+\sum_{j=1}^{m} \sum_{i=1}^{n}\left|L_{1 j} \alpha_{i j}^{1}\right| \int_{0}^{+\infty} e^{\varepsilon s} K_{1 j}(s)\left|V_{j}(t-s)\right|^{2} d s \\
= & \sum_{i=1}^{n} \sum_{j=1}^{m}\left|L_{1 j} \alpha_{i j}^{1}\right| \check{K}_{1 j}(\varepsilon)\left|U_{i}(t)\right|^{2}+\sum_{j=1}^{m} \sum_{i=1}^{n}\left|L_{1 j} \alpha_{i j}^{1}\right| \check{K}_{1 j}(\varepsilon)\left|V_{j}(t)\right|^{2} \\
& -\sum_{j=1}^{m} \sum_{i=1}^{n}\left|L_{1 j} \alpha_{i j}^{1}\right| \frac{d}{d t} \int_{0}^{+\infty} e^{\varepsilon s} K_{1 j}(s) \int_{t-s}^{t}\left|V_{j}(\theta)\right|^{2} d \theta d s,
\end{aligned}
$$

where the second inequality follows from Lemma 2.2, the third inequality follows from the Cauchy-Schwarz inequality, and the equality follows from some routine calculations. Similarly, we have

$$
\begin{aligned}
2 \mid \sum_{i=1}^{n} e^{\varepsilon t} U_{i}(t)\left[\bigvee_{j=1}^{m} \beta_{i j}^{1} \int_{-\infty}^{t} K_{1 j}(t-s) f_{1 j}\left(v_{j}^{*}+e^{-\varepsilon s} V_{j}(s)\right) d s\right. \\
\left.\quad-\bigvee_{j=1}^{m} \beta_{i j}^{1} \int_{-\infty}^{t} K_{1 j}(t-s) f_{1 j}\left(v_{j}^{*}\right) d s\right] \mid \\
\leq \sum_{i=1}^{n} \sum_{j=1}^{m}\left|L_{1 j} \beta_{i j}^{1}\right| \check{K}_{1 j}(\varepsilon)\left|U_{i}(t)\right|^{2}+\sum_{j=1}^{m} \sum_{i=1}^{n}\left|L_{1 j} \beta_{i j}^{1}\right| \check{K}_{1 j}(\varepsilon)\left|V_{j}(t)\right|^{2} \\
\quad-\sum_{j=1}^{m} \sum_{i=1}^{n}\left|L_{1 j} \beta_{i j}^{1}\right| \frac{d}{d t} \int_{0}^{+\infty} e^{\varepsilon s} K_{1 j}(s) \int_{t-s}^{t}\left|V_{j}(\theta)\right|^{2} d \theta d s .
\end{aligned}
$$

We further have

$$
\begin{aligned}
2 \mid & \sum_{i=1}^{n} \mu_{1 i} e^{\varepsilon \tau_{1 i}}\left(\mu_{1 i} e^{\varepsilon \tau_{1 i}}-\varepsilon\right) U_{i}(t) \int_{t-\tau_{1 i}}^{t} U_{i}(s) d s \mid \\
& \leq 2 \sum_{i=1}^{n} \mu_{1 i} e^{\varepsilon \tau_{1 i}}\left(\mu_{1 i} e^{\varepsilon \tau_{1 i}}-\varepsilon\right)\left|U_{i}(t)\right| \int_{t-\tau_{1 i}}^{t}\left|U_{i}(s)\right| d s \\
& \leq \sum_{i=1}^{n} \mu_{1 i} e^{\varepsilon \tau_{1 i}}\left(\mu_{1 i} e^{\varepsilon \tau_{1 i}}-\varepsilon\right) \int_{t-\tau_{1 i}}^{t}\left(\left|U_{i}(s)\right|^{2}+\left|U_{i}(t)\right|^{2}\right) d s \\
& =2 \sum_{i=1}^{n} \tau_{1 i} \mu_{1 i} e^{\varepsilon \tau_{1 i}}\left(\mu_{1 i} e^{\varepsilon \tau_{1 i}}-\varepsilon\right)\left|U_{i}(t)\right|^{2} \\
& \quad-\sum_{i=1}^{n} \mu_{1 i} e^{\varepsilon \tau_{1 i}}\left(\mu_{1 i} e^{\varepsilon \tau_{1 i}}-\varepsilon\right) \frac{d}{d t} \int_{t-\tau_{1 i}}^{t}\left|U_{i}(s)\right|^{2} d s,
\end{aligned}
$$

where the second inequality follows from the Cauchy-Schwarz inequality, and the equality follows from some routine calculations. We have

$$
\begin{aligned}
& 2\left|\sum_{i=1}^{n} \sum_{j=1}^{m} \mu_{1 i} e^{\varepsilon \tau_{1 i}} a_{i j}^{1} e^{\varepsilon t} \int_{t-\tau_{1 i}}^{t} U_{i}(s) d s\left(f_{1 j}\left(v_{j}^{*}+e^{-\varepsilon t} V_{j}(t)\right)-f_{1 j}\left(v_{j}^{*}\right)\right)\right| \\
& \quad \leq 2 \sum_{i=1}^{n} \sum_{j=1}^{m} \mu_{1 i} e^{\varepsilon \tau_{1 i}}\left|L_{1 j} a_{i j}^{1}\right| \int_{t-\tau_{1 i}}^{t}\left|U_{i}(s)\right| d s\left|V_{j}(t)\right|
\end{aligned}
$$




$$
\begin{aligned}
\leq & \sum_{i=1}^{n} \sum_{j=1}^{m} \mu_{1 i} e^{\varepsilon \tau_{1 i}}\left|L_{1 j} a_{i j}^{1}\right| \int_{t-\tau_{1 i}}^{t}\left|U_{i}(s)\right|^{2} d s+\sum_{j=1}^{m} \sum_{i=1}^{n} \mu_{1 i} e^{\varepsilon \tau_{1 i}} \tau_{1 i}\left|L_{1 j} a_{i j}^{1}\right|\left|V_{j}(t)\right|^{2} \\
= & \sum_{i=1}^{n} \sum_{j=1}^{m} \mu_{1 i} e^{\varepsilon \tau_{1 i}}\left|L_{1 j} a_{i j}^{1}\right|\left|U_{i}(s)\right|^{2} d s+\sum_{j=1}^{m} \sum_{i=1}^{n} \mu_{1 i} e^{\varepsilon \tau_{1 i}} \tau_{1 i}\left|L_{1 j} a_{i j}^{1}\right|\left|V_{j}(t)\right|^{2} \\
& -\sum_{i=1}^{n} \sum_{j=1}^{m} \mu_{1 i} e^{\varepsilon \tau_{1 i}}\left|L_{1 j} a_{i j}^{1}\right| \frac{d}{d t} \int_{t-\tau_{1 i}}^{t} \int_{\tau}^{t}\left|U_{i}(s)\right|^{2} d s d \tau,
\end{aligned}
$$

where the first inequality follows from the triangle inequality, the second inequality follows from the Cauchy-Schwarz inequality, and the equality follows from some routine calculations. By some calculations as in (3.8) and (3.12), we obtain

$$
\begin{aligned}
2 \mid \sum_{i=1}^{n} & \sum_{j=1}^{m} \mu_{1 i} e^{\varepsilon \tau_{1 i}} b_{i j}^{1} e^{\varepsilon t} \int_{t-\tau_{1 i}}^{t} U_{i}(s) d s\left(f_{1 j}\left(v_{j}^{*}+e^{-\varepsilon\left(t-\sigma_{1 j}(t)\right)} V_{j}\left(t-\sigma_{1 j}(t)\right)\right)-f_{1 j}\left(v_{j}^{*}\right)\right) \mid \\
\leq & \sum_{i=1}^{n} \sum_{j=1}^{m} \mu_{1 i} e^{\varepsilon \tau_{1 i}} e^{\varepsilon \bar{\sigma}_{1 j}}\left|L_{1 j} b_{i j}^{1}\right| \tau_{1 i}\left|U_{i}(t)\right|^{2} \\
& -\sum_{i=1}^{n} \sum_{j=1}^{m} \mu_{1 i} e^{\varepsilon \tau_{1 i}} e^{\varepsilon \bar{\sigma}_{1 j}}\left|L_{1 j} b_{i j}^{1}\right| \frac{d}{d t} \int_{t-\tau_{1 i}}^{t} \int_{\tau}^{t}\left|U_{i}(s)\right|^{2} d s d \tau \\
& +\sum_{j=1}^{m} \sum_{i=1}^{n} \frac{\mu_{1 i} e^{\varepsilon \tau_{1 i}} e^{\varepsilon \bar{\sigma}_{1 j}} \tau_{1 i}\left|L_{1 j} b_{i j}^{1}\right|\left(\dot{\sigma}_{1 j}-\hat{\sigma}_{1 j}\right)}{1-\hat{\sigma}_{1 j}}\left|V_{j}\left(t-\sigma_{1 j}(t)\right)\right|^{2} \\
& -\sum_{j=1}^{m} \sum_{i=1}^{n} \frac{\mu_{1 i} e^{\varepsilon \tau_{1 i}}\left|b_{i j}^{1}\right| L_{1 j} e^{\varepsilon \bar{\sigma}_{1 j}} \tau_{1 i}}{1-\hat{\sigma}_{1 j}} \frac{d}{d t} \int_{t-\sigma_{1 j}(t)}^{t}\left|V_{j}(s)\right|^{2} d s \\
& +\sum_{j=1}^{m} \sum_{i=1}^{n} \frac{\mu_{1 i} e^{\varepsilon \tau_{1 i}}\left|b_{i j}^{1}\right| L_{1 j} e^{\varepsilon \bar{\sigma}_{1 j}} \tau_{1 i} \bar{\sigma}_{1 j}}{1-\hat{\sigma}_{1 j}}\left|V_{j}(t)\right|^{2} .
\end{aligned}
$$

We have

$$
\begin{aligned}
2 \mid \sum_{i=1}^{n} \mu_{1 i} e^{\varepsilon \tau_{1 i}} e^{\varepsilon t} \int_{t-\tau_{1 i}}^{t} U_{i}(s) d s\left[\bigwedge_{j=1}^{m} \alpha_{i j}^{1} \int_{-\infty}^{t} K_{1 j}(t-s) f_{1 j}\left(v_{j}^{*}+e^{-\varepsilon s} V_{j}(s)\right) d s\right. \\
\left.\quad-\bigwedge_{j=1}^{m} \alpha_{i j}^{1} \int_{-\infty}^{t} K_{1 j}(t-s) f_{1 j}\left(v_{j}^{*}\right) d s\right] \mid \\
\leq 2 \sum_{i=1}^{n} \sum_{j=1}^{m} \mu_{1 i} e^{\varepsilon \tau_{1 i}}\left|L_{1 j} \alpha_{i j}^{1}\right| \int_{t-\tau_{1 i}}^{t}\left|U_{i}(s)\right| d s \int_{-\infty}^{t} e^{\varepsilon(t-s)} K_{1 j}(t-s)\left|V_{j}(s)\right| d s \\
\leq \sum_{i=1}^{n} \sum_{j=1}^{m} \mu_{1 i} e^{\varepsilon \tau_{1 i}}\left|L_{1 j} \alpha_{i j}^{1}\right| \check{K}_{1 j}(\varepsilon) \int_{t-\tau_{1 i}}^{t}\left|U_{i}(s)\right|^{2} d s \\
\quad+\sum_{j=1}^{m} \sum_{i=1}^{n} \mu_{1 i} e^{\varepsilon \tau_{1 i}} \tau_{1 i}\left|L_{1 j} \alpha_{i j}^{1}\right| \int_{0}^{+\infty} e^{\varepsilon s} K_{1 j}(s)\left|V_{j}(t-s)\right|^{2} d s \\
=\sum_{i=1}^{n} \sum_{j=1}^{m} \mu_{1 i} e^{\varepsilon \tau_{1 i}}\left|L_{1 j} \alpha_{i j}^{1}\right| \check{K}_{1 j}(\varepsilon) \tau_{1 i}\left|U_{i}(t)\right|^{2}
\end{aligned}
$$




$$
\begin{aligned}
& -\sum_{i=1}^{n} \sum_{j=1}^{m} \mu_{1 i} e^{\varepsilon \tau_{1 i}}\left|L_{1 j} \alpha_{i j}^{1}\right| \check{K}_{1 j}(\varepsilon) \frac{d}{d t} \int_{t-\tau_{1 i}}^{t} \int_{\tau}^{t}\left|U_{i}(s)\right|^{2} d s d \tau \\
& -\sum_{j=1}^{m} \sum_{i=1}^{n} \mu_{1 i} e^{\varepsilon \tau_{1 i}} \tau_{1 i}\left|L_{1 j} \alpha_{i j}^{1}\right| \frac{d}{d t} \int_{0}^{+\infty} e^{\varepsilon s} K_{1 j}(s) \int_{t-s}^{t}\left|V_{j}(\theta)\right|^{2} d \theta d s \\
& +\sum_{j=1}^{m} \sum_{i=1}^{n} \mu_{1 i} e^{\varepsilon \tau_{1 i}} \tau_{1 i}\left|L_{1 j} \alpha_{i j}^{1}\right| \check{K}_{1 j}(\varepsilon)\left|V_{j}(t)\right|^{2},
\end{aligned}
$$

where the first inequality follows from Lemma 2.2, the second inequality follows from the Cauchy-Schwarz inequality, and the equality follows from some routine calculations. Similarly, we have

$$
\begin{aligned}
2 \mid \sum_{i=1}^{n} \mu_{1 i} e^{\varepsilon \tau_{1 i}} e^{\varepsilon t} \int_{t-\tau_{1 i}}^{t} U_{i}(s) d s \\
\times\left[\bigvee_{j=1}^{m} \beta_{i j}^{1} \int_{-\infty}^{t} K_{1 j}(t-s) f_{1 j}\left(v_{j}^{*}+e^{-\varepsilon s} V_{j}(s)\right) d s\right. \\
\left.\quad-\bigvee_{j=1}^{m} \beta_{i j}^{1} \int_{-\infty}^{t} K_{1 j}(t-s) f_{1 j}\left(v_{j}^{*}\right) d s\right] \mid \\
\leq \sum_{j=1}^{m} \sum_{i=1}^{n} \mu_{2 j} e^{\varepsilon \tau_{2 j} j}\left|L_{2 i} \alpha_{j i}^{2}\right| \check{K}_{2 i}(\varepsilon) \tau_{2 j}\left|V_{j}(t)\right|^{2} \\
\quad-\sum_{j=1}^{m} \sum_{i=1}^{n} \mu_{2 j} e^{\varepsilon \tau_{2 j}}\left|L_{2 i} \alpha_{j i}^{2}\right| \check{K}_{2 i}(\varepsilon) \frac{d}{d t} \int_{t-\tau_{2 j}}^{t} \int_{\tau}^{t}\left|V_{j}(s)\right|^{2} d s d \tau \\
\quad-\sum_{i=1}^{n} \sum_{j=1}^{m} \mu_{2 j} e^{\varepsilon \tau_{2 j}} \tau_{2 j}\left|L_{2 i} \alpha_{j i}^{2}\right| \frac{d}{d t} \int_{0}^{+\infty} e^{\varepsilon s} K_{2 i}(s) \int_{t-s}^{t}\left|U_{i}(\theta)\right|^{2} d \theta d s \\
\quad+\sum_{i=1}^{n} \sum_{j=1}^{m} \mu_{2 j} e^{\varepsilon \tau_{2 j}} \tau_{2 j}\left|L_{2 i} \alpha_{j i}^{2}\right| \check{K}_{2 i}(\varepsilon)\left|U_{i}(t)\right|^{2} .
\end{aligned}
$$

Now, we estimate the term $\sum_{i=1}^{n}\left|\mathfrak{u}_{2 i}(t)\right|^{2}$. By the inequality $(a+b+c+d)^{2} \leq 4\left(a^{2}+b^{2}+\right.$ $c^{2}+d^{2}$ ) we have

$$
\begin{aligned}
\sum_{i=1}^{n}\left|\mathfrak{u}_{2 i}(t)\right|^{2} \leq & 4 \sum_{i=1}^{n}\left|\sum_{j=1}^{m} \tilde{a}_{i j}^{1} e^{\varepsilon t}\left(\tilde{f}_{1 j}\left(v_{j}^{*}+e^{-\varepsilon t} V_{j}(t)\right)-\tilde{f}_{1 j}\left(v_{j}^{*}\right)\right)\right|^{2} \\
& +4 \sum_{i=1}^{n}\left|\sum_{j=1}^{m} \tilde{b}_{i j}^{1} e^{\varepsilon t}\left(\tilde{f}_{1 j}\left(v_{j}^{*}+e^{-\varepsilon\left(t-\tilde{\alpha}_{1 j}(t)\right)} V_{j}\left(t-\tilde{\sigma}_{1 j}(t)\right)\right)-\tilde{f}_{1 j}\left(v_{j}^{*}\right)\right)\right|^{2} \\
& +4 \sum_{i=1}^{n} \mid \bigwedge_{j=1}^{m} \tilde{\alpha}_{i j}^{1} e^{\varepsilon t} \int_{-\infty}^{t} \tilde{K}_{1 j}(t-s) \tilde{f}_{1 j}\left(v_{j}^{*}+e^{-\varepsilon s} V_{j}(s)\right) d s \\
& -\left.\bigwedge_{j=1}^{m} \tilde{\alpha}_{i j}^{1} e^{\varepsilon t} \int_{-\infty}^{t} \tilde{K}_{1 j}(t-s) \tilde{f}_{1 j}\left(v_{j}^{*}\right) d s\right|^{2}
\end{aligned}
$$




$$
\begin{aligned}
& +4 \sum_{i=1}^{n} \mid \bigvee_{j=1}^{m} \tilde{\beta}_{i j}^{1} e^{\varepsilon t} \int_{-\infty}^{t} \tilde{K}_{1 j}(t-s) \tilde{f}_{1 j}\left(v_{j}^{*}+e^{-\varepsilon s} V_{j}(s)\right) d s \\
& -\left.\bigvee_{j=1}^{m} \tilde{\beta}_{i j}^{1} e^{\varepsilon t} \int_{-\infty}^{t} \tilde{K}_{1 j}(t-s) \tilde{f}_{1 j}\left(v_{j}^{*}\right) d s\right|^{2} .
\end{aligned}
$$

We have

$$
\begin{aligned}
& \sum_{i=1}^{n}\left|\sum_{j=1}^{m} \tilde{a}_{i j}^{1} e^{\varepsilon t}\left(\tilde{f}_{1 j}\left(v_{j}^{*}+e^{-\varepsilon t} V_{j}(t)\right)-\tilde{f}_{1 j}\left(v_{j}^{*}\right)\right)\right|^{2} \\
& \quad \leq \sum_{i=1}^{n}\left(\sum_{j=1}^{m}\left|\tilde{a}_{i j}^{1}\right| \tilde{L}_{1 j}\left|V_{j}(t)\right|\right)^{2} \\
& \quad \leq\left(\sum_{i=1}^{n} \sum_{j=1}^{m}\left|\tilde{L}_{1 j} \tilde{a}_{i j}^{1}\right|^{2}\right) \sum_{j=1}^{m}\left|V_{j}(t)\right|^{2}
\end{aligned}
$$

where the first inequality follows from the triangle inequality and the definition of $\tilde{L}_{1 j}$, and the second inequality follows from the Cauchy-Schwarz inequality. By mimicking the steps in (3.8) we have

$$
\begin{aligned}
\sum_{i=1}^{n} \mid & \left|\sum_{j=1}^{m} \tilde{b}_{i j}^{1} e^{\varepsilon t}\left(\tilde{f}_{1 j}\left(v_{j}^{*}+e^{-\varepsilon\left(t-\tilde{\sigma}_{1 j}(t)\right)} V_{j}\left(t-\tilde{\sigma}_{1 j}(t)\right)\right)-\tilde{f}_{1 j}\left(v_{j}^{*}\right)\right)\right|^{2} \\
\leq & \sum_{j=1}^{m} \sum_{i=1}^{n}\left|\tilde{L}_{1 j} \tilde{b}_{i j}^{1}\right|^{2} e^{2 \varepsilon \tilde{\sigma}_{1 j}} \sum_{j=1}^{m}\left|V_{j}\left(t-\tilde{\sigma}_{1 j}(t)\right)\right|^{2} \\
= & \left(\sum_{j=1}^{m} \sum_{i=1}^{n} \frac{\left|\tilde{b}_{i j}^{1}\right|^{2}\left|\tilde{L}_{1 j}\right|^{2}\left(\dot{\tilde{\sigma}}_{1 j}(t)-\hat{\tilde{\sigma}}_{1 j}\right)}{1-\hat{\tilde{\sigma}}_{1 j}}\right) \sum_{j=1}^{m}\left|V_{j}\left(t-\tilde{\sigma}_{1 j}(t)\right)\right|^{2} \\
& -\left(\sum_{j=1}^{m} \sum_{i=1}^{n} \frac{\left|\tilde{b}_{i j}^{1}\right|^{2}\left|\tilde{L}_{1 j}\right|^{2}}{1-\hat{\tilde{\sigma}}_{1 j}}\right) \sum_{j=1}^{m} \frac{d}{d t} \int_{t-\tilde{\sigma}_{1 j}(t)}^{t}\left|V_{j}(s)\right|^{2} d s \\
& +\left(\sum_{j=1}^{m} \sum_{i=1}^{n} \frac{\left|\tilde{b}_{i j}^{1}\right|^{2}\left|\tilde{L}_{1 j}\right|^{2} \overline{\tilde{\sigma}}_{1 j}}{1-\hat{\tilde{\sigma}}_{1 j}}\right) \sum_{j=1}^{m}\left|V_{j}(t)\right|^{2},
\end{aligned}
$$

where the inequality follows from the triangle inequality and the definition of $\tilde{L}_{1 j}$. We have

$$
\begin{aligned}
\sum_{i=1}^{n} \mid \bigwedge_{j=1}^{m} \tilde{\alpha}_{i j}^{1} e^{\varepsilon t} \int_{-\infty}^{t} \tilde{K}_{1 j}(t-s) \tilde{f}_{1 j}\left(v_{j}^{*}+e^{-\varepsilon s} V_{j}(s)\right) d s \\
\quad-\left.\bigwedge_{j=1}^{m} \tilde{\alpha}_{i j}^{1} e^{\varepsilon t} \int_{-\infty}^{t} \tilde{K}_{1 j}(t-s) \tilde{f}_{1 j}\left(v_{j}^{*}\right) d s\right|^{2} \\
\leq \sum_{i=1}^{n}\left[\sum_{j=1}^{m} e^{\varepsilon t}\left|\tilde{\alpha}_{i j}^{1}\right| \mid \int_{-\infty}^{t} \tilde{K}_{1 j}(t-s) \tilde{f}_{1 j}\left(v_{j}^{*}+e^{-\varepsilon s} V_{j}(s)\right) d s\right. \\
\left.\quad-\int_{-\infty}^{t} \tilde{K}_{1 j}(t-s) \tilde{f}_{1 j}\left(v_{j}^{*}\right) d s \mid\right]^{2}
\end{aligned}
$$




$$
\begin{aligned}
\leq & \sum_{i=1}^{n}\left[\sum_{j=1}^{m}\left|\tilde{L}_{1 j} \tilde{\alpha}_{i j}^{1}\right| \int_{-\infty}^{t} e^{\varepsilon(t-s)} \tilde{K}_{1 j}(t-s)\left|V_{j}(s)\right| d s\right]^{2} \\
\leq & \sum_{i=1}^{n}\left[\sum_{j=1}^{m}\left|\tilde{L}_{1 j} \tilde{\alpha}_{i j}^{1}\right|\left(\check{\tilde{K}}_{1 j}(\varepsilon) \int_{0}^{+\infty} e^{\varepsilon s} \tilde{K}_{1 j}(s)\left|V_{j}(t-s)\right|^{2} d s\right)^{\frac{1}{2}}\right]^{2} \\
\leq & \sum_{i=1}^{n} \sum_{j=1}^{m}\left|\tilde{L}_{1 j} \tilde{\alpha}_{i j}^{1}\right|^{2}\left[\sum_{j=1}^{m} \check{\tilde{K}}_{1 j}(\varepsilon) \int_{0}^{+\infty} e^{\varepsilon s} \tilde{K}_{1 j}(s)\left|V_{j}(t-s)\right|^{2} d s\right] \\
= & \sum_{j=1}^{m} \check{\tilde{K}}_{1 j}(\varepsilon)\left(\sum_{i=1}^{n} \sum_{j=1}^{m}\left|\tilde{L}_{1 j} \tilde{\alpha}_{i j}^{1}\right|^{2} \check{\tilde{K}}_{1 j}(\varepsilon)\right)\left|V_{j}(t)\right|^{2} \\
& -\left(\sum_{i=1}^{n} \sum_{j=1}^{m}\left|\tilde{L}_{1 j} \tilde{\alpha}_{i j}^{1}\right|^{2} \check{\tilde{K}}_{1 j}(\varepsilon)\right) \sum_{j=1}^{m} \frac{d}{d t} \int_{0}^{+\infty} e^{\varepsilon s} \tilde{K}_{1 j}(s) \int_{t-s}^{t}\left|V_{j}(\theta)\right|^{2} d \theta d s,
\end{aligned}
$$

where the second inequality follows from the Lemma 2.2, the third inequality (resp., fourth) follows from the Cauchy-Schwarz inequality for functions (resp., for finite sequences), and the equality follows from some routine calculations. Similarly, we have

$$
\begin{aligned}
\sum_{i=1}^{n} \mid \bigvee_{j=1}^{m} \tilde{\beta}_{i j}^{1} e^{\varepsilon t} \int_{-\infty}^{t} \tilde{K}_{1 j}(t-s) \tilde{f}_{1 j}\left(v_{j}^{*}+e^{-\varepsilon s} V_{j}(s)\right) d s \\
\quad-\left.\bigvee_{j=1}^{m} \tilde{\beta}_{i j}^{1} e^{\varepsilon t} \int_{-\infty}^{t} \tilde{K}_{1 j}(t-s) \tilde{f}_{1 j}\left(v_{j}^{*}\right) d s\right|^{2} \\
\leq \sum_{j=1}^{m}\left|\check{\tilde{K}}_{1 j}(\varepsilon)\right|^{2}\left(\sum_{i=1}^{n} \sum_{j=1}^{m}\left|\tilde{L}_{1 j} \tilde{\beta}_{i j}^{1}\right|^{2}\right)\left|V_{j}(t)\right|^{2} \\
\quad-\sum_{j=1}^{m} \check{\tilde{K}}_{1 j}(\varepsilon)\left(\sum_{i=1}^{n} \sum_{j=1}^{m}\left|\tilde{L}_{1 j} \tilde{\beta}_{i j}^{1}\right|^{2}\right) \frac{d}{d t} \int_{0}^{+\infty} e^{\varepsilon s} \tilde{K}_{1 j}(s) \int_{t-s}^{t}\left|V_{j}(\theta)\right|^{2} d \theta d s .
\end{aligned}
$$

Combining (3.4), (3.5), (3.6), (3.7), (3.8), (3.9), (3.10), (3.11), (3.12), (3.13), (3.14), (3.15), (3.16), (3.17), (3.18), (3.19), (3.20) and utilizing the symmetry of $U_{i}(t)$ and $V_{j}(t)$, by some calculations we obtain

$$
\begin{aligned}
& d\left[\mathcal{V}_{1}(t ; \varepsilon)+\mathcal{V}_{2}(t ; \varepsilon)+\mathcal{V}_{3}(t ; \varepsilon)+\mathcal{V}_{4}(t ; \varepsilon)\right] \\
& \leq \sum_{i=1}^{n} M_{1 i}(\varepsilon)\left|U_{i}(t)\right|^{2} d t+\sum_{j=1}^{m} M_{2 j}(\varepsilon)\left|V_{j}(t)\right|^{2} d t \\
& \quad+\sum_{i=1}^{n} \sum_{j=1}^{m} \frac{\left|b_{j i}^{2}\right| L_{2 i} e^{\varepsilon \bar{\sigma}_{2 i}\left(\mu_{2 j} e^{\varepsilon \tau_{2 j}}+1\right)\left(\dot{\sigma}_{2 i}(t)-\hat{\sigma}_{2 i}\right)}\left|U_{i}\left(t-\sigma_{2 i}(t)\right)\right|^{2} d t}{1-\hat{\sigma}_{2 i}}\left|V_{j}\left(t-\sigma_{1 j}(t)\right)\right|^{2} d t \\
& \left.\quad+\sum_{j=1}^{m} \sum_{i=1}^{n} \frac{\left|b_{i j}^{1}\right| L_{1 j} e^{\varepsilon \bar{\sigma}_{1 j}}\left(\mu_{1 i} e^{\varepsilon \tau_{1 i}}+1\right)\left(\dot{\sigma}_{1 j}(t)-\hat{\sigma}_{1 j}\right)}{1-\hat{\sigma}_{1 j}}\right] \sum_{i=1}^{n}\left|U_{i}\left(t-\tilde{\sigma}_{2 i}(t)\right)\right|^{2} d t \\
& \quad+4\left[\sum_{i=1}^{n} \sum_{j=1}^{m} \frac{\left|\tilde{b}_{j i}^{2}\right|^{2}\left|\tilde{L}_{2 i}\right|^{2}\left(\dot{\tilde{\sigma}}_{2 i}(t)-\hat{\tilde{\sigma}}_{2 i}\right)}{1-\hat{\tilde{\sigma}}_{2 i}}\right.
\end{aligned}
$$




$$
\begin{aligned}
& +4\left[\sum_{j=1}^{m} \sum_{i=1}^{n} \frac{\left|\tilde{b}_{i j}^{1}\right|^{2}\left|\tilde{L}_{1 j}\right|^{2}\left(\dot{\tilde{\sigma}}_{1 j}(t)-\hat{\tilde{\sigma}}_{1 j}\right)}{1-\hat{\tilde{\sigma}}_{1 j}}\right] \sum_{j=1}^{m}\left|V_{j}\left(t-\tilde{\sigma}_{1 j}(t)\right)\right|^{2} d t \\
& +2 \sum_{i=1}^{n}\left[U_{i}(t)-\mu_{1 i} e^{\varepsilon \tau_{1 i}} \int_{t-\tau_{1 i}}^{t} U_{i}(s) d s\right] \mathfrak{a}_{2 i}(t) d B(t) \\
& +2 \sum_{j=1}^{m}\left[V_{j}(t)-\mu_{1 i} e^{\varepsilon \tau_{2 j}} \int_{t-\tau_{2 j}}^{t} V_{j}(s) d s\right] \mathfrak{b}_{2 j}(t) d B(t) \\
& \leq \sum_{i=1}^{n} M_{1 i}(\varepsilon)\left|U_{i}(t)\right|^{2} d t+\sum_{j=1}^{m} M_{2 j}(\varepsilon)\left|V_{j}(t)\right|^{2} d t \\
& +2 \sum_{i=1}^{n}\left[U_{i}(t)-\mu_{1 i} e^{\varepsilon \tau_{1 i}} \int_{t-\tau_{1 i}}^{t} U_{i}(s) d s\right] \mathfrak{a}_{2 i}(t) d B(t) \\
& +2 \sum_{j=1}^{m}\left[V_{j}(t)-\mu_{1 i} e^{\varepsilon \tau_{2 j}} \int_{t-\tau_{2 j}}^{t} V_{j}(s) d s\right] \mathfrak{b}_{2 j}(t) d B(t), \\
& \forall t \in[0,+\infty), \mathbb{P} \text {-a.s. }
\end{aligned}
$$

Therefore

$$
\frac{d}{d t} \mathcal{V}(t ; \varepsilon) \leq \sum_{i=1}^{n} M_{1 i}(\varepsilon) \mathbb{E}\left|U_{i}(t)\right|^{2}+\sum_{j=1}^{m} M_{2 j}(\varepsilon) \mathbb{E}\left|V_{j}(t)\right|^{2}, \quad \forall t \in[0,+\infty)
$$

Since $M_{1 i}(\varepsilon)$ and $M_{2 j}(\varepsilon)$ are continuous and $M_{1 i}(0)<0$ and $M_{2 j}(0)<0$, there exists $\varepsilon^{*} \in$ $(0, \bar{\varepsilon})$ such that $M_{1 i}\left(\varepsilon^{*}\right) \leq 0$ and $M_{2 j}\left(\varepsilon^{*}\right) \leq 0$, and therefore $\tilde{\mathcal{V}}\left(t ; \varepsilon^{*}\right)$ is decreasing. This, together with the definition of $\tilde{\mathcal{V}}\left(t ; \varepsilon^{*}\right)$, directly implies

$$
\begin{aligned}
& \mathbb{E} \sum_{i=1}^{n}\left|U_{i}(t)-\mu_{1 i} e^{\varepsilon \tau_{1 i}} \int_{t-\tau_{1 i}}^{t} U_{i}(s) d s\right|^{2}+\mathbb{E} \sum_{j=1}^{m}\left|V_{j}(t)-\mu_{2 j} e^{\varepsilon \tau_{2 j}} \int_{t-\tau_{2 j}}^{t} V_{j}(s) d s\right|^{2} \\
& \quad \leq \mathcal{V}\left(t ; \varepsilon^{*}\right) \leq \mathcal{V}\left(0 ; \varepsilon^{*}\right), \quad \forall t \in[0,+\infty) .
\end{aligned}
$$

Applying the inequality $(a+b)^{2} \leq 2\left(a^{2}+b^{2}\right)$, we have

$$
\begin{aligned}
\mathbb{E}\left(\sum_{i=1}^{n}\left|U_{i}(t)\right|^{2}+\sum_{j=1}^{m}\left|V_{j}(t)\right|^{2}\right) \\
\leq 2 \mathbb{E} \sum_{i=1}^{n}\left|U_{i}(t)-\mu_{1 i} e^{\varepsilon_{0} \tau_{1 i}} \int_{t-\tau_{1 i}}^{t} U_{i}(s) d s\right|^{2}+2 \mathbb{E} \sum_{i=1}^{n}\left|\mu_{1 i} e^{\varepsilon_{0} \tau_{1 i}} \int_{t-\tau_{1 i}}^{t} U_{i}(s) d s\right|^{2} \\
\quad+2 \mathbb{E} \sum_{j=1}^{m}\left|V_{j}(t)-\mu_{2 j} e^{\varepsilon_{0} \tau_{2 j}} \int_{t-\tau_{2 j}}^{t} V_{j}(s) d s\right|^{2}+2 \mathbb{E} \sum_{j=1}^{m}\left|\mu_{2 j} e^{\varepsilon_{0} \tau_{2 j}} \int_{t-\tau_{2 j}}^{t} V_{j}(s) d s\right|^{2} \\
\leq 2 \mathcal{V}\left(0 ; \varepsilon^{*}\right)+2 \max \left(\left(\mu_{1 i}\right)^{2} e^{2 \varepsilon_{0} \tau_{1 i} i} \tau_{1 i},\left(\mu_{2 j}\right)^{2} e^{2 \varepsilon_{0} \tau_{2 j}} \tau_{2 j}\right) \\
\quad \times \int_{t-\max \left(\bigvee_{i=1}^{n} \tau_{1 i}, \bigvee_{j=1}^{m} \tau_{2 j}\right)}^{t} \sum_{i=1}^{n} \mathbb{E}\left(\sum_{i=1}^{n}\left|U_{i}(t)\right|^{2}+\sum_{j=1}^{m}\left|V_{j}(t)\right|^{2}\right) d s, \quad \forall t \in[0,+\infty) .
\end{aligned}
$$


By Gronwall's lemma we have

$$
\mathbb{E}\left(\sum_{i=1}^{n}\left|U_{i}(t)\right|^{2}+\sum_{j=1}^{m}\left|V_{j}(t)\right|^{2}\right) \leq C, \quad \forall t \in[0,+\infty)
$$

or equivalently

$$
\mathbb{E}\left(\sum_{i=1}^{n}\left|u_{i}(t)-u_{i}^{*}\right|^{2}+\sum_{j=1}^{m}\left|v_{j}(t)-v_{j}^{*}\right|^{2}\right) \leq A e^{-2 \varepsilon_{0} t}, \quad \forall t \in[0,+\infty)
$$

where

$$
\begin{aligned}
A & =2 \mathcal{V}\left(0 ; \varepsilon^{*}\right) \exp \left(\max \left(\left(\mu_{1 i}\right)^{2} e^{2 \varepsilon_{0} \tau_{1 i}} \tau_{1 i},\left(\mu_{2 j}\right)^{2} e^{2 \varepsilon_{0} \tau_{2 j}} \tau_{2 j}\right) \max \left(\bigvee_{i=1}^{n} \tau_{1 i}, \bigvee_{j=1}^{m} \tau_{2 j}\right)\right) \\
& \leq \tilde{A} \sup _{s \in(-\infty, 0]} \mathbb{E}\left|\left(\phi_{1}(\cdot, s), \ldots, \phi_{n}(\cdot, s), \psi_{1}(\cdot, s), \ldots, \psi_{m}(\cdot, s)\right)\right|^{2},
\end{aligned}
$$

where $\tilde{A}$ is independent of the initial data $\left(\phi_{1}, \ldots, \phi_{n}, \psi_{1}, \ldots, \psi_{m}\right)$. The proof is complete.

\section{An illustrative example}

In this section, by using the aforeobtained results we attempt to investigate the meansquare stability of the following system:

$$
\begin{aligned}
& \begin{aligned}
d u_{1}(t)=[ & -32 u_{1}\left(t-\frac{1}{2048}\right)+\sin (v(t))+\sin \left(v\left(\frac{2 t^{2}+t}{2 t+2}\right)\right) \\
& +\int_{-\infty}^{t} e^{-(t-s)} \sin (v(s)) d s
\end{aligned} \\
& \left.+c_{11}^{1} w_{1}^{1}+T_{11}^{1} w_{11}^{1}+H_{11}^{1} w_{11}^{1}+I_{1}\right] d t \\
& +\left[\cos (v(t))+\cos \left(v\left(\frac{2 t^{2}+t}{2 t+2}\right)\right)\right. \\
& \left.+\int_{-\infty}^{t} e^{-(t-s)} \cos (v(s)) d s\right] d B(t), \\
& d u_{2}(t)=\left[-32 u_{2}\left(t-\frac{1}{2048}\right)+\sin (v(t))+\sin \left(v\left(\frac{2 t^{2}+t}{2 t+2}\right)\right)\right. \\
& +\int_{-\infty}^{t} e^{-(t-s)} \sin (v(s)) d s \\
& \left.+c_{21}^{1} w_{1}^{1}+T_{21}^{1} w_{21}^{1}+H_{21}^{1} w_{21}^{1}+I_{2}\right] d t \\
& +\left[\cos (v(t))+\cos \left(v\left(\frac{2 t^{2}+t}{2 t+2}\right)\right)\right. \\
& \left.+\int_{-\infty}^{t} e^{-(t-s)} \cos (v(s)) d s\right] d B(t), \\
& d v(t)=\left[-32 v\left(t-\frac{1}{2048}\right)+\cos \left(u_{1}(t)\right)+\sin \left(u_{2}(t)\right)\right. \\
& +\cos \left(u_{1}\left(\frac{2 t^{2}+t}{2 t+2}\right)\right)+\sin \left(u_{2}\left(\frac{2 t^{2}+t}{2 t+2}\right)\right) \int_{-\infty}^{t} e^{-(t-s)} \cos \left(u_{1}(s)\right) d s \\
& +\int_{-\infty}^{t} e^{-(t-s)} \sin \left(u_{2}(s)\right) d s \\
& \left.+\sum_{i=1}^{2} c_{1 i}^{2} w_{i}^{2}+\bigwedge_{i=1}^{2} T_{1 i}^{2} w_{1 i}^{2}+\bigvee_{i=1}^{2} H_{1 i}^{2} w_{1 i}^{2}+J\right] d t \\
& +\left[\tanh \left(u_{1}(t)\right)+\arctan \left(u_{2}(t)\right)+\tanh \left(u_{1}\left(\frac{2 t^{2}+t}{2 t+2}\right)\right)\right. \\
& +\arctan \left(u_{2}\left(\frac{2 t^{2}+t}{2 t+2}\right)\right)+\int_{-\infty}^{t} e^{-(t-s)} \tanh \left(u_{1}(s)\right) d s \\
& \left.+\int_{-\infty}^{t} e^{-(t-s)} \arctan \left(u_{2}(s)\right) d s\right] d B(t) \text {. }
\end{aligned}
$$

We can recast this system into the form of (2.1) with $\tau_{11}=\tau_{12}=\tau_{21}=\frac{1}{2048}, \mu_{11}=\mu_{12}=$ $\mu_{21}=32, f_{11}(u)=\sin u, \tilde{f}_{11}(u)=\cos u, f_{21}(u)=\cos u, f_{22}(u)=\sin u, \tilde{f}_{21}(u)=\tanh u=\frac{e^{x}-e^{-x}}{e^{x}+e^{-x}}$, 
$\tilde{f}_{22}(u)=\arctan u, L_{11}=\tilde{L}_{11}=L_{21}=L_{22}=\tilde{L}_{21}=\tilde{L}_{22}=1, \sigma_{11}(t)=\sigma_{21}(t)=\sigma_{22}(t)=\tilde{\sigma}_{11}(t)=$ $\tilde{\sigma}_{21}(t)=\tilde{\sigma}_{22}(t)=\frac{t}{2 t+2}, K_{11}(t)=\tilde{K}_{11}(t)=K_{21}(t)=\tilde{K}_{21}(t)=K_{21}(t)=\tilde{K}_{22}(t)=e^{-t}, \check{K}_{11}(0)=$ $\check{\tilde{K}}_{11}(0)=\check{K}_{21}(0)=\check{\tilde{K}}_{21}(0)=\check{K}_{21}(0)=\check{\tilde{K}}_{22}(0)=1, a_{11}^{1}=a_{21}^{1}=b_{11}^{1}=b_{21}^{1}=\alpha_{11}^{1}=\alpha_{21}^{1}=\beta_{11}^{1}=$ $\beta_{21}^{1}=\tilde{a}_{11}^{1}=\tilde{a}_{21}^{1}=\tilde{b}_{11}^{1}=\tilde{b}_{21}^{1}=\tilde{\alpha}_{11}^{1}=\tilde{\alpha}_{21}^{1}=\tilde{\beta}_{11}^{1}=\tilde{\beta}_{21}^{1}=a_{11}^{2}=a_{12}^{2}=b_{11}^{2}=b_{12}^{2}=\alpha_{11}^{2}=\beta_{12}^{2}=\tilde{a}_{11}^{2}=$ $\tilde{a}_{12}^{2}=\tilde{b}_{11}^{2}=\tilde{b}_{12}^{2}=\tilde{\alpha}_{11}^{2}=\tilde{\beta}_{12}^{2}=1, \alpha_{12}^{2}=\beta_{11}^{2}=\tilde{\alpha}_{12}^{2}=\tilde{\beta}_{11}^{2}=0$, and $\bar{\sigma}_{11}=\bar{\sigma}_{21}=\bar{\sigma}_{22}=\overline{\tilde{\sigma}}_{11}=\overline{\tilde{\sigma}}_{21}=$ $\overline{\tilde{\sigma}}_{22}=\hat{\sigma}_{11}=\hat{\sigma}_{21}=\hat{\sigma}_{22}=\hat{\tilde{\sigma}}_{11}=\hat{\tilde{\sigma}}_{21}=\hat{\tilde{\sigma}}_{22}=\frac{1}{2}$. Then we have

$$
\begin{aligned}
M_{1 i}(0)= & -2 \mu_{1 i}+\left|a_{i 1}^{1}\right| L_{11}+\left|a_{1 i}^{2}\right| L_{2 i}+\left|b_{i 1}^{1}\right| L_{11}+\left|\alpha_{i 1}^{1}\right| L_{11} \check{K}_{11}(0)+\left|\alpha_{1 i}^{2}\right| L_{2 i} \check{K}_{2 i}(0) \\
& +\left|\beta_{i 1}^{1}\right| L_{11} \check{K}_{11}(0)+\left|\beta_{1 i}^{2}\right| L_{2 i} \check{K}_{2 i}(0)+2\left(\mu_{1 i}\right)^{2} \tau_{1 i}+\frac{\left|b_{1 i}^{2}\right| L_{2 i} \bar{\sigma}_{2 i}}{1-\hat{\sigma}_{2 i}}+\mu_{1 i}\left|a_{i 1}^{1}\right| L_{11} \tau_{1 i} \\
& +\mu_{21}\left|a_{1 i}^{2}\right| L_{2 i} \tau_{21}+\mu_{1 i}\left|b_{i 1}^{1}\right| L_{11} \tau_{1 i}+\frac{\mu_{21}\left|b_{1 i}^{2}\right| L_{2 i} \tau_{21} \bar{\sigma}_{2 i}}{1-\hat{\sigma}_{2 i}}+\left|\alpha_{i 1}^{1}\right| L_{11} \mu_{1 i} \check{K}_{11}(0) \tau_{1 i} \\
& +\left|\alpha_{1 i}^{2}\right| L_{2 i} \mu_{21} \check{K}_{2 i}(0) \tau_{21}+4 \sum_{i=1}^{2}\left|\tilde{a}_{1 i}^{2}\right|^{2}\left|\tilde{L}_{2 i}\right|^{2}+4 \sum_{i=1}^{2} \frac{\left|\tilde{b}_{1 i}^{2}\right|^{2}\left|\tilde{L}_{2 i}\right|^{2} \overline{\tilde{\sigma}}_{2 i}}{1-\tilde{\tilde{\sigma}}_{2 i}} \\
& +4\left(\sum_{i=1}^{2}\left|\tilde{\alpha}_{1 i}^{2}\right|^{2}\left|\tilde{L}_{2 i}\right|^{2} \check{\tilde{K}}_{2 i}(0)\right) \check{\tilde{K}}_{2 i}(0)+4\left(\sum_{i=1}^{2}\left|\tilde{\beta}_{1 i}^{2}\right|^{2}\left|\tilde{L}_{2 i}\right|^{2} \check{\check{K}}_{2 i}(0)\right) \check{\tilde{K}}_{2 i}(0) \\
= & -22.9063
\end{aligned}
$$

and

$$
\begin{aligned}
M_{21}(0)= & -2 \mu_{21}+\sum_{i=1}^{2}\left|a_{i 1}^{1}\right| L_{11}+\sum_{i=1}^{2}\left|a_{1 i}^{2}\right| L_{2 i}+\sum_{i=1}^{2} \frac{\left|b_{i 1}^{1}\right| L_{11} \bar{\sigma}_{11}}{1-\hat{\sigma}_{11}} \\
& +\sum_{i=1}^{2}\left|\alpha_{i 1}^{1}\right| L_{11} \check{K}_{11}(0)+\sum_{i=1}^{2}\left|\alpha_{1 i}^{2}\right| L_{2 i} \check{K}_{2 i}(0)+\sum_{i=1}^{2}\left|\beta_{i 1}^{1}\right| L_{11} \check{K}_{11}(0) \\
& +\sum_{i=1}^{2}\left|\beta_{1 i}^{2}\right| L_{2 i} \check{K}_{2 i}(0)+2\left(\mu_{21}\right)^{2} \tau_{21}+\sum_{i=1}^{2}\left|b_{1 i}^{2}\right| L_{2 i}+\sum_{i=1}^{2} \mu_{1 i}\left|a_{i 1}^{1}\right| L_{11} \tau_{1 i} \\
& +\sum_{i=1}^{2} \mu_{21}\left|a_{1 i}^{2}\right| L_{2 i} \tau_{21}+\sum_{i=1}^{2} \frac{\mu_{1 i}\left|b_{i 1}^{1}\right| L_{11} \tau_{1 i} \bar{\sigma}_{11}}{1-\hat{\sigma}_{11}}+\sum_{i=1}^{2} \mu_{21}\left|b_{1 i}^{2}\right| L_{2 i} \tau_{21} \\
& +\sum_{i=1}^{2}\left|\alpha_{i 1}^{1}\right| L_{11} \mu_{1 i} \check{K}_{11}(0) \tau_{1 i}+\sum_{i=1}^{2}\left|\alpha_{1 i}^{2}\right| L_{2 i} \mu_{21} \check{K}_{2 i}(0) \tau_{21}+4 \sum_{i=1}^{2}\left|\tilde{a}_{i 1}^{1}\right|^{2}\left|\tilde{L}_{11}\right|^{2} \\
& +4 \sum_{i=1}^{2} \frac{\left|\tilde{b}_{i 1}^{1}\right|^{2}\left|\tilde{L}_{11}\right|^{2} \overline{\tilde{\sigma}}_{11}}{1-\tilde{\tilde{\sigma}}_{11}}+4\left(\sum_{i=1}^{2}\left|\tilde{\alpha}_{1 i}^{2}\right|^{2}\left|\tilde{L}_{2 i}\right|^{2} \check{\tilde{K}}_{11}(0)\right) \check{\tilde{K}}_{11}(0) \\
& +4\left(\sum_{i=1}^{2}\left|\tilde{\beta}_{1 i}^{2}\right|^{2}\left|\tilde{L}_{2 i}\right|^{2} \check{\tilde{K}}_{11}(0)\right) \check{\tilde{K}}_{11}(0)=-12.8125
\end{aligned}
$$

Therefore by Theorem 3.2 we have that system (4.1) is mean-square exponentially stable.

\section{Conclusions}

We proved in the paper that fuzzy stochastic BAMNs with delays are mean-square exponentially stable, provided that the amplification effect of the activation functions is weak 
enough and that the transmission coefficients are small enough. Our study is worth some additional remarks:

- As indicated in the introduction, the deterministic BAMNs have received extensive and intensive investigations. One of the most striking phenomenons in this direction is that a large number of experts have constructed very elaborate Lyapunov-Krasovskii functions to reduce the conservatism of the stability results. Note, however, that just a few of these ideas can be applied to study the stability problems for stochastic systems. Therefore, it is extremely promising to work in the direction to prove less conservative stability results for stochastic neural networks.

- For the stochastic dynamical systems, it is equally (or even more) interesting to study the $p$ th moment (exponential) stability. Therefore, we shall contemplate, in the next step, more carefully the structure of the neural networks in the paper and try our best to obtain the $p$ th moment exponential stability results.

- More realistically, some dissipative mechanism, in one form or another, should be introduced into neural networks, and thus we are led to the so-called reaction-diffusion neural networks (RDNNs). In the past two decades, RDNNs have received extensive attentions. Inspired by the results obtained in these studies, we are tempted to study fuzzy stochastic reaction-diffusion BAM neural networks for their long-time behavior.

Acknowledgements

Fosheng is supported by the Initial Foundation of Mianyang Teachers' College (Grant No. QD2016A003). Chengqiang is supported by NSFC (\#11701050 and \#11571244), by JG Program (\#2017JG13) of Chengdu Normal University, and by SCJYT Program (\#18ZB0098) of Sichuan Province, China.

\section{Funding}

Fosheng is supported by the Initial Foundation of Mianyang Teachers' College (Grant No. QD2016A003). Chengqiang is supported by NSFC (\#11701050 and \#11571244), by JG Program (\#2017JG13) of Chengdu Normal University, and by SCJYT Program (\#18ZB0098) of Sichuan Province, China.

\section{Competing interests}

The authors declare that they have no competing interests.

Authors' contributions

The authors contributed to the work totally, and they read and approved the final version of the manuscript.

\section{Author details}

'School of Mathematics and Physics, Mianyang Teachers' College, Mianyang, China. ${ }^{2}$ School of Mathematics, Chengdu Normal University, Chengdu, China.

\section{Publisher's Note}

Springer Nature remains neutral with regard to jurisdictional claims in published maps and institutional affiliations.

Received: 4 March 2018 Accepted: 30 May 2018 Published online: 09 July 2018

\section{References}

1. Wang, Z.S., Liu, Z.W., Zheng, C.D.: Qualitative Analysis and Control of Complex Neural Networks with Delays. Science Press, Beijing (2015)

2. Kosko, B.: Adaptive bi-directional associative memories. Appl. Opt. 26(23), 4947-4960 (1987)

3. Kosko, B.: Bi-directional associative memories. IEEE Trans. Syst. Man Cybern. 18(1), $49-60$ (1988)

4. Li, L., Jian, J.: Exponential $p$-convergence analysis for stochastic BAM neural networks with time-varying and infinite distributed delays. Appl. Math. Comput. 266, 860-873 (2015)

5. Gopalsamy, K.: Leakage delays in BAM. J. Math. Anal. Appl. 325, 1117-1132 (2007)

6. Liu, B.W.: Global exponential stability for BAM neural networks with time-varying delays in the leakage terms. Nonlinear Anal., Real World Appl. 14(1), 559-566 (2013)

7. Duan, L.D., Huang, L.H.: Global exponential stability of fuzzy BAM neural networks with distributed delays and time-varying delays in the leakage terms. Neural Comput. Appl. 23, 171-178 (2013)

8. Cai, Z.W., Huang, L.H.: Functional differential inclusions and dynamic behaviors for memristor-based BAM neural networks with time-varying delays. Commun. Nonlinear Sci. Numer. Simul. 19(5), 1279-1300 (2014) 
9. Wang, F., Liu, M.C.: Global exponential stability of high-order bidirectional associative memory (BAM) neural networks with time delays in leakage terms. Neurocomputing 177, 515-528 (2016)

10. Balasubramaniam, P., Kalpana, M., Rakkiyappan, R.: Global asymptotic stability of BAM fuzzy cellular neural networks with time delay in the leakage term, discrete and unbounded distributed delays. Math. Comput. Model. 53(5-6), 839-853 (2011)

11. Li, Y.K., Fan, X.L.: Existence and globally exponential stability of almost periodic solution for Cohen-Grossberg BAM neural networks with variable coefficients. Appl. Math. Model. 33(4), 2114-2120 (2009)

12. Song, Q.K., Zhao, Z.J.: Stability criterion of complex-valued neural networks with both leakage delay and time-varying delays on time scales. Neurocomputing 171, 179-184 (2016)

13. Song, Q.K., Cao, J.D.: Exponential stability for impulsive BAM neural networks with time-varying delays and reaction-diffusion terms. Adv. Differ. Equ. 2017, 78160 (2007)

14. Xu, C.J., Zhang, Q.M., Wu, Y.S.: Existence and stability of pseudo almost periodic solutions for shunting inhibitory cellular neural networks with neutral type delays and time-varying leakage delays. Netw. Comput. Neural Syst. 25(4), 168-192 (2014)

15. Xu, C.J., Li, P.L.: Existence and exponentially stability of anti-periodic solutions for neutral BAM neural networks with time-varying delays in the leakage terms. J. Nonlinear Sci. Appl. 9(3), 1285-1305 (2016)

16. Li, Y.K., Wang, C.: Existence and global exponential stability of equilibrium for discrete-time fuzzy BAM neural networks with variable delays and impulses. Fuzzy Sets Syst. 217, 62-79 (2013)

17. Zhang, Z.Q., Liu, K.Y.: Existence and global exponential stability of a periodic solution to interval general bidirectional associative memory (BAM) neural networks with multiple delays on time scales. Neural Netw. 24(5), 427-439 (2011)

18. Berezansky, L., Braverman, E., Idels, L.: New global exponential stability criteria for nonlinear delay differential systems with applications to BAM neural networks. Appl. Math. Comput. 243, 899-910 (2014)

19. Zhang, Z.Q., Liu, W.B., Zhou, D.M.: Global asymptotic stability to a generalized Cohen-Grossberg BAM neural networks of neutral type delays. Neural Netw. 25, 94-105 (2012)

20. Li, X.D.: Exponential stability of Cohen-Grossberg-type BAM neural networks with time-varying delays via impulsive control. Neurocomputing 73(1-3), 525-530 (2009)

21. Rakkiyappan, R., Lakshmanan, S., Sivasamy, R., Lim, C.P.: Leakage-delay-dependent stability analysis of Markovian jumping linear systems with time-varying delays and nonlinear perturbations. Appl. Math. Model. 40(7-8), 5026-5043 (2016)

22. Li, X.D., Fu, X.L., Balasubramanianm, P., Rakkiyappan, R.: Existence, uniqueness and stability analysis of recurrent neural networks with time delay in the leakage term under impulsive perturbations. Nonlinear Anal., Real World Appl. 11, 4092-4108 (2011)

23. Balasubramanianm, P., Vembarasan, V., Rakkiyappan, R.: Leakage delay in T-S fuzzy cellular neural networks. Neural Process. Lett. 33, 111-136 (2011)

24. Li, Y.K., Yang, L., Sun, L.J.: Existence and exponential stability of an equilibrium point for fuzzy BAM neural networks with time-varying delays in leakage terms on time scales. Adv. Differ. Equ. 2013, 218 (2013)

25. Xu, C.J., Li, P.L.: Exponential stability for fuzzy BAM cellular neural networks with distributed leakage delays and impulses. Adv. Differ. Equ. 2016, 276 (2016)

26. Li, Y.K., Li, Y.Q.: Exponential stability of BAM fuzzy cellular neural networks with time-varying delays in leakage terms and impulses. Abstr. Appl. Anal. 2014, Article ID 634394 (2014)

27. Xu, C., Chen, L., Guo, T., Li, P.: Dynamics of FCNNs with proportional delays and leakage delays. Adv. Differ. Equ. 2018 $72(2018)$

28. Xu, C., Li, P.: Global exponential convergence of fuzzy cellular neural networks with leakage delays, distributed delays and proportional delays. Circuits Syst. Signal Process. 37(1), 163-177 (2018)

29. Xu, C., Li, P., Pang, Y.: Existence and global exponential stability of almost periodic solutions for BAM neural networks with distributed leakage delays on time scales. J. Appl. Anal. Comput. 7(4), 1200-1232 (2017)

30. Xu, C., Li, P.: $p$ th moment exponential stability of stochastic fuzzy Cohen-Grossberg neural networks with discrete and distributed delays. Nonlinear Anal. 22(4), 531-544 (2017)

31. Xu, C., Li, P.: Global exponential convergence of neutral-type Hopfield neural networks with multi-proportional delays and leakage delays. Chaos Solitons Fractals 96, 139-144 (2017)

32. Xu, C., Zhang, Q.: On antiperiodic solutions for Cohen-Grossberg shunting inhibitory neural networks with time-varying delays and impulses. Neural Comput. 26(10), 2328-2349 (2014)

33. Xu, C., Li, P., Pang, Y.: Exponential stability of almost periodic solutions for memristor-based neural networks with distributed leakage delays. Neural Comput. 28(12), 1-31 (2016)

34. Xu, C., Li, P.: Periodic dynamics for memristor-based bidirectional associative memory neural networks with leakage delays and time-varying delays. Int. J. Control. Autom. Syst. 16(2), 535-549 (2018)

35. Sakthivel, R., Anbuvithya, R., Mathiyalagan, K., Ma, Y.K., Prakash, P.: Reliable anti-synchronization conditions for BAM memristive neural networks with different memductance functions. Appl. Math. Comput. 275, 213-228 (2016)

36. Anbuvithya, R., Mathiyalagan, K., Sakthivel, R., Prakash, P.: Passivity of memristor-based BAM neural networks with different memductance and uncertain delays. Cogn. Neurodyn. 10(4), 339-351 (2016)

37. Mathiyalagan, K., Anbuvithya, R., Sakthivel, R., Ju, H.P., Prakash, P.: Non-fragile $H_{\infty}$ synchronization of memristor-based neural networks using passivity theory. Neural Netw. 74, 85-100 (2016)

38. Zhu, Q.X., Rakkiyappan, R., Chandrasekar, A.: Stochastic stability of Markovian jump BAM neural networks with leakage delays and impulse control. Neurocomputing 136, 136-151 (2014)

39. Senthilraj, S., Raja, R., Zhu, Q.X., Samidurai, R., Yao, Z.S.: Exponential passivity analysis of stochastic neural networks with leakage, distributed delays and Markovian jumping parameters. Neurocomputing 175, 401-410 (2016)

40. Balasubramaniam, P., Vidhya, C.: Global asymptotic stability of stochastic BAM neural networks with distributed delays and reaction-diffusion terms. J. Comput. Appl. Math. 234(12), 3458-3466 (2010)

41. Zhu, Q., Li, X., Yang, X.: Exponential stability for stochastic reaction-diffusion BAM neural networks with time-varying and distributed delays. Appl. Math. Comput. 217(13), 6078-6091 (2011)

42. Li, X., Fu, X.: Global asymptotic stability of stochastic Cohen-Grossberg-type BAM neural networks with mixed delays: an LMI approach. J. Comput. Appl. Math. 235(12), 3385-3394 (2011) 
43. Bao, H., Cao, J.: Exponential stability for stochastic BAM networks with discrete and distributed delays. Appl. Math. Comput. 218(11), 6188-6199 (2012)

44. Rakkiyappan, R., Chandrasekar, A., Lakshmanan, S., Park, J.H.: Exponential stability for Markovian jumping stochastic BAM neural networks with mode-dependent probabilistic time-varying delays and impulse control. Complexity 20(3), 39-65 (2015)

45. Ye, Z., Zhang, H., Zhang, H., Zhang, H., Lu, G.: Mean square stabilization and mean square exponential stabilization of stochastic BAM neural networks with Markovian jumping parameters. Chaos Solitons Fractals 73, 156-165 (2015)

46. Syed Ali, M., Balasubramaniam, P., Rihan, F.A., Lakshmanan, S.: Stability criteria for stochastic Takagi-Sugeno fuzzy Cohen-Grossberg BAM neural networks with mixed time-varying delays. Complexity 21(5), 143-154 (2016)

47. Rao, R., Wang, X., Zhong, S.: LMI-based stability criterion for impulsive delays Markovian jumping time-delays reaction-diffusion BAM neural networks via Gronwall-Bellman-type impulsive integral inequality. Math. Probl. Eng. 2015, Article ID 185854 (2015)

48. Syed Ali, M., Balasubramaniam, P.: Robust stability for uncertain stochastic fuzzy BAM neural networks with time-varying delays. Phys. Lett. A 372(31), 5159-5166 (2008)

49. Vidhya, C., Balasubramaniam, P.: Robust stability of uncertain Markovian jumping stochastic Cohen-Grossberg type BAM neural networks with time-varying delays and reaction diffusion terms. Neural Parallel Sci. Comput. 19(1-2), 181-195 (2011)

50. Mathiyalagan, K., Sakthivel, R., Anthoni, S.M.: New robust passivity criteria for stochastic fuzzy BAM neural networks with time-varying delays. Commun. Nonlinear Sci. Numer. Simul. 17(3), 1392-1407 (2012)

51. Sakthivel, R., Raja, R., Anthoni, S.M.: Linear matrix inequality approach to stochastic stability of uncertain delayed BAM neural networks. IMA J. Appl. Math. 78(6), 1156-1178 (2013)

52. Pan, T.T., Shi, B., Yang, S.J., Zhang, Q.: Stability analysis of stochastic BAM-type Cohen-Grossberg neural networks with delays and impulses. Acta Math. Sci. Ser. A Chin. Ed. 33(5), 937-950 (2013)

53. Rao, R.F., Zhong, S.M., Wang, X.R.: Stochastic stability criteria with LMI conditions for Markovian jumping impulsive BAM neural networks with mode-dependent time-varying delays and nonlinear reaction-diffusion. Commun. Nonlinear Sci. Numer. Simul. 19(1), 258-273 (2014)

54. Du, Y., Zhong, S., Zhou, N.: Global asymptotic stability of Markovian jumping stochastic Cohen-Grossberg BAM neural networks with discrete and distributed time-varying delays. Appl. Math. Comput. 243, 624-636 (2014)

\section{Submit your manuscript to a SpringerOpen ${ }^{\circ}$ journal and benefit from:}

- Convenient online submission

- Rigorous peer review

- Open access: articles freely available online

- High visibility within the field

- Retaining the copyright to your article

Submit your next manuscript at $\boldsymbol{~ s p r i n g e r o p e n . c o m ~}$ 